\title{
EXPANSIONS IN TERMS OF HEAT POLYNOMIALS AND ASSOCIATED FUNCTIONS
}

BY

P. C. ROSENBLOOM AND D. V. WIDDER(1)

Introduction. A heat polynomial $v_{n}(x, t)$ of degree $n$ is defined as the coefficient of $z^{n} / n$ ! in the power series expansion $e^{z x+z^{2} t}$ :

$$
e^{z x+z^{2} t}=\sum_{n=0}^{\infty} v_{n}(x, t) \frac{z^{n}}{n !} .
$$

It is clearly a solution of the heat equation

$$
\frac{\partial^{2} u}{\partial x^{2}}=\frac{\partial u}{\partial t}
$$

for all values of the variables. An associated function $w_{n}(x, t)$ is defined as

$$
w_{n}(x, t)=k(x, t) v_{n}\left(\frac{x}{t}, \frac{-1}{t}\right),
$$

where $k(x, t)$ is the source solution or fundamental solution of $(2)$ :

$$
k(x, t)=\frac{e^{-x^{2} / 4 t}}{(4 \pi t)^{1 / 2}} .
$$

Thus $w_{n}(x, t)$ is obtained from $v_{n}(x, t)$ by the familiar Appell transformation and is consequently itself a solution of (2). Alternatively $w_{n}(x, t)$ may be defined by use of the fundamental solution itself as a generating function by a series analogous to (1):

$$
k(x-2 z, t)=\sum_{n=0}^{\infty} w_{n}(x, t) \frac{z^{n}}{n !} .
$$

Hence

$$
w_{n}(x, t)=\left.\frac{\partial^{n}}{\partial z^{n}} k(x-2 z, t)\right|_{z=0} .
$$

It is shown that the heat polynomials and associated functions form a bi-

Presented to the Society, April 19, 1958 under the title Representation of solutions of the heat equation; received by the editors January 15, 1958.

(1) The research of the first author was supported in part by the Office of Naval Research under Contract No. Nonr-710(16); the research of the second author was supported in part by the United States Air Force under Contract No. AF 18(600)-1461. 
orthogonal set in the following sense:

$$
\int_{-\infty}^{\infty} v_{n}(x,-t) w_{n}(x, t) d x=\delta_{m n} \quad 0<t<\infty .
$$

We are thus led to consider solutions of (2) which have one or the other of the expansions

$$
\begin{aligned}
& u(x, t)=\sum_{n=0}^{\infty} a_{n} v_{n}(x, t), \\
& u(x, t)=\sum_{n=0}^{\infty} b_{n} w_{n}(x, t) .
\end{aligned}
$$

The coefficients may be determined in the obvious way by use of (3) but they also have other useful determinations. In fact, both may be expressed in terms of derivatives as in the case of Taylor's series. For (4) we have

$$
a_{n}=\frac{1}{n !} \frac{\partial^{n} u}{\partial x^{n}}(0,0)
$$

for (5) see formula (6) below. A relation that is basic in the study of these series is

$$
k(x-y, s-t)=\sum_{n=0}^{\infty} \frac{v_{n}(x,-t) w_{n}(y, s)}{n ! 2^{n}} .
$$

The domain of convergence of a series of type (4) is found to be a strip $|t|<\sigma$; that for (4) a half plane $t>\sigma$. The extent of these domains can be determined, as in Taylor's series, by function theoretic properties of the sum of the series. Let us outline briefly how this is done.

We say that $u(x, t) \in H^{*}$ in a strip $a<t<b$ if it satisfies (2) and has the Huygens property there; that is,

$$
u(x, t)=\int_{-\infty}^{\infty} k\left(x-y, t-t^{\prime}\right) u\left(y, t^{\prime}\right) d y
$$

for every pair $t, t^{\prime}$ such that $a<t^{\prime}<t<b$. Membership in class $H^{*}$ is to expansions (4) what analyticity is to Taylor expansions. For, $u(x, t)$ has a development (4) in the largest strip $|t|<\sigma$ for which $u(x, t) \in H^{*}$.

There is a dual result for series (5). In fact $u(x, t)$ has such a development if and only if

$$
u(x, t)=\frac{1}{2 \pi} \int_{-\infty}^{\infty} e^{i x y-t y^{2}} \phi(y) d y,
$$

and it is function theoretic properties of $\phi(y)$ (its order and type as an entire function) which determine the extent of the validity of (5). Moreover, 


$$
b_{n}=\frac{\phi^{(n)}(0)}{n !(-2 i)^{n}} .
$$

The known existence of null solutions of $(2)$ (solutions $\not \equiv 0$ which vanish identically on a line $t=$ constant) makes it evident that the Huygens property cannot be enjoyed by all solutions. Hence it is useful to have a working criterion for the class $H^{*}$. Here is a very practical one: $u(x, t) \in H^{*}$ in $a<t<b$ if it satisfies (2) and if

$$
\int_{-\infty}^{\infty}|u(x, t)| k\left(x, b^{\prime}-t\right) d x
$$

is uniformly bounded on $a^{\prime} \leqq t \leqq b^{\prime}$ for every pair $\left(a^{\prime}, b^{\prime}\right), a<a^{\prime}<b^{\prime}<b$.

The $L^{2}$ convergence theory of the series (4) and (5) is also studied. The Parseval relation in particular yields interesting power series in the time variable. Many illustrative examples are given in the last section.

1. Heat polynomials. We define $v_{n}(x, t)$ as a polynomial of degree $n$, homogeneous in $x$ and $t^{1 / 2}$, with coefficient of $x^{n}$ equal to unity, and satisfying the heat equation

$$
\frac{\sigma^{2} u}{\partial x^{2}}=\frac{\partial u}{\partial t}
$$

for all $x$ and $t$. We shall call $v_{n}(x, t)$ the heat polynomial of degree $n$. It is uniquely determined and is in fact

$$
v_{n}(x, t)=n ! \sum_{k=0}^{[n / 2]} \frac{x^{n-2 k}}{(n-2 k) !} \frac{t^{k}}{k !} .
$$

In particular

$v_{0}=1, \quad v_{1}=x, \quad v_{2}=x^{2}+2 t, \quad v_{3}=x^{3}+6 x t, \quad v_{4}=x^{4}+12 x^{2} t+12 t^{2}$.

Note that $v_{n}(x, t)$ is even or odd with $n$, as a function of $x$. Since the coefficients (1.2) are positive $v_{2 n}(x, t)$ is always positive for $t>0$ and $v_{n}(x, t)>0$ for $x>0$ and $t>0$.

Let us next obtain the Poisson representation

$$
v_{n}(x, t)=\int_{-\infty}^{\infty} k(x-y, t) y^{n} d y=k(x, t) * x^{n} \quad t>0,
$$

where $k(x, t)$ is the source solution or fundamental solution of (1.1):

$$
k(x, t)=\frac{e^{-x^{2} / 4 t}}{(4 \pi t)^{1 / 2}} \quad t>0 .
$$

The integral (1.3) is equal to 


$$
\int_{-\infty}^{\infty} k(y, t) \sum_{k=0}^{n}\left(\begin{array}{l}
n \\
k
\end{array}\right) x^{n-k}(-y)^{k} d y=\int_{-\infty}^{\infty} k(y, t) \sum_{k=0}^{[n / 2]}\left(\begin{array}{c}
n \\
2 k
\end{array}\right) x^{n-2 k} y^{2 k} d y .
$$

But

$$
\int_{-\infty}^{\infty} k(y, t) y^{2 k} d y=\frac{(4 t)^{k}}{(\pi)^{1 / 2}} \int_{0}^{\infty} e^{-y} y^{k-1 / 2} d y=\frac{(2 k) ! t^{k}}{k !} .
$$

Substituting (1.5) in (1.4) we obtain (1.3) as stated.

For future reference we record that

$$
v_{2 n+1}(0, t)=0, \quad v_{2 n}(0, t)=\frac{(2 n) !}{n !} t^{n} .
$$

Let us next derive a generating function for the heat polynomials. Using (1.3) we have for $t>0$

$$
\sum_{n=0}^{\infty} \frac{z^{n}}{n !} v_{n}(x, t)=\sum_{n=0}^{\infty} \frac{z^{n}}{n !} k(x, t) * x^{n}=k(x, t) * e^{x z} .
$$

This exchange of integral and summation signs is valid for all complex $z$ and for $-\infty<x<\infty, 0<t<\infty$ since

$$
\int_{-\infty}^{\infty} e^{-(x-y)^{2} / 4 t} e^{|y z|} d y<\infty .
$$

Moreover, it is well known that

$$
k(x, t) * e^{x z}=e^{t D^{2}} e^{x z}=e^{x z+t z^{2}} .
$$

Here the symbolic operator $e^{t D^{2}}$, where $D$ stands for differentiation with respect to $x$, serves as a tool for the rapid recovery of such results as (1.7). We have proved

$$
e^{x z+t z^{2}}=\sum_{n=0}^{\infty} \frac{z^{n}}{n !} v_{n}(x, t) \quad-\infty<x<\infty, 0<t<\infty
$$

for all complex $z$.

We easily derive from (1.8) that

$$
\frac{\partial}{\partial x} v_{n}(x, t)=n v_{n-1}(x, t), \quad \frac{\partial}{\partial t} v_{n}(x, t)=n(n-1) v_{n-2}(x, t) .
$$

The heat polynomials are closely related to Hermite polynomials. We adopt the notation

$$
\begin{aligned}
H_{n}(x) & =(-1)^{n} e^{x^{2}} D^{n} e^{-x^{2}}, \\
e^{2 z x-z^{2}} & =\sum_{n=0}^{\infty} \frac{z^{n}}{n !} H_{n}(x) .
\end{aligned}
$$


Comparing (1.8) with (1.10) we have

$$
v_{n}(x, t)=(-t)^{n / 2} H_{n}\left(x /(-4 t)^{1 / 2}\right) \quad-\infty<x<\infty,-\infty<t<\infty .
$$

We recall that the set of functions

$$
e^{-x^{2}} x^{n}
$$

$$
n=0,1,2, \cdots,
$$

is closed in $L$ or in $L^{2}$ on the interval $(-\infty, \infty)$. By (1.11) this shows that the set

$$
k(x, t) v_{n}(x,-t) \quad n=0,1,2, \cdots
$$

is closed in $L$ or in $L^{2}$ in $-\infty<x<\infty$ for each fixed $t>0$. That is, if $f(x)$ is in either class and is such that

$$
\int_{-\infty}^{\infty} f(x) k(x, t) v_{n}(x,-t) d x=0 \quad n=0,1,2, \cdots
$$

for any fixed $t>0$, then $f(x)$ is zero almost everywhere.

2. The associated functions. Introduce the following notation for solutions of (1.1).

Definition 2.1. A function $u(x, t) \in H$ in a domain $D$ if and only if $u(x, t) \in C^{2}$ and satisfies Equation (1.1) there.

We shall call functions of class $H$ temperature functions. There is a familiar transformation due to P. Appell [1, p. 187] which carries functions of $H$ into others of the class. Thus if $u(x, t) \in H$ in $D$ then $k(x, t) u(x / t,-1 / t) \in H$ when $(x / t,-1 / t)$ is in $D$. We apply this transformation to the heat polynomials (1.2), adopting the following notation.

Definition 2.1. For $n=0,1,2, \cdots$

$$
w_{n}(x, t)=k(x, t) v_{n}(x / t,-1 / t) \quad 0<t<\infty .
$$

We call this set of functions the set associated with the set of heat polynomials.

By the homogeneity of $v_{n}(x, t)$ we have $v_{n}\left(\lambda x,-\lambda^{2} t\right)=\lambda^{n} v_{n}(x,-t)$, or for $\lambda=1 / t$

$$
v_{n}(x / t,-1 / t)=t^{-n} v_{n}(x,-t)
$$

Hence

$$
w_{n}(x, t)=k(x, t) v_{n}(x,-t) t^{-n} .
$$

Equation (2.1) enables us to obtain from (1.8) a generating function for the associated set:

$$
\begin{aligned}
\sum_{n=0}^{\infty} \frac{z^{n}}{n ! 2^{n}} w_{n}(x, t) & =k(x, t) \sum_{n=0}^{n} \frac{v_{n}(x,-t)}{n !}\left(\frac{z}{2 t}\right)^{n} \\
& =k(x-z, t),
\end{aligned}
$$

or 


$$
k(x-2 z, t)=\sum_{n=0}^{\infty} \frac{z^{n}}{n !} w_{n}(x, t) \quad-\infty<x<\infty, 0<t<\infty
$$

for all complex $z$. Comparing this with the Maclaurin expansion

$$
k(x-2 z, t)=\sum_{n=0}^{\infty} k^{(n)}(x, t) \frac{(-2 z)^{n}}{n !},
$$

we obtain

$$
w_{n}(x, t)=(-2)^{n} k^{(n)}(x, t)
$$$$
n=0,1,2, \cdots .
$$

Here, and always, the superscript indicates differentiation with respect to the first argument. If we differentiate (2.3) we obtain

$$
w_{n}^{\prime}(x, t)=-\frac{1}{2} w_{n+1}(x, t) \text {. }
$$

But since

$$
k^{\prime}(x-2 z, t)=-\frac{(x-2 z)}{2 t} k(x-2 z, t),
$$

an alternative expression for $w_{n}^{\prime}(x, t)$ is available:

$$
2 t w_{n}^{\prime}(x, t)=-x w_{n}(x, t)+2 n w_{n-1}(x, t), \quad n=1,2, \cdots .
$$

This yields the recursion formula

$$
t w_{n+1}(x, t)=x w_{n}(x, t)-2 n w_{n-1}(x, t), \quad n=1,2, \cdots .
$$

A formula analogous to (1.11) is obtained from (2.1)

$$
w_{n}(x, t)=t^{-n / 2} k(x, t) H_{n}\left(\frac{x}{(4 t)^{1 / 2}}\right) .
$$

And now the familiar orthogonality relations for the Hermite polynomials,

$$
\begin{aligned}
(\pi)^{1 / 2} \int_{-\infty}^{\infty} e^{-x^{2}} H_{m}(x) H_{n}(x) d x & =\delta_{m, n} c_{n}, \\
c_{n} & =n ! 2^{n}
\end{aligned}
$$

shows that the set $w_{n}(x, t)$ is biorthogonal to the set $v_{n}(x, t)$ :

$$
\int_{-\infty}^{\infty} w_{m}(x, t) v_{n}(x,-t) d x=\delta_{m n} c_{n} \quad 0<t<\infty .
$$

From (1.12) we see that the set $w_{n}(x, t), n=0,1, \cdots$, is closed in $L$ or $L^{2}$ on $-\infty<x<\infty$ for each fixed $t>0$.

3. Asymptotic estimates. For further study it is essential to know the behavior of the functions $v_{n}(x, t)$ and $w_{n}(x, t)$ for large $n$. For negative $t$ this 
can be derived from the known behavior of the Hermite polynomials. However, for $t>0$ the argument of $H_{n}$ in formula (1.11) is purely imaginary. Available asymptotic formulas in this case are inadequate for our purposes, and we make independent estimates.

We first recall the following formula, H. Bateman [2, p. 201],

$$
\begin{aligned}
\Gamma\left(\frac{n}{2}+1\right) e^{-x^{2} / 2} H_{n}(x) & \\
& =\Gamma(n+1)\left[\cos \left((2 n+1)^{1 / 2} x-\frac{n \pi}{2}\right)+O\left(1 /(n)^{1 / 2}\right)\right],
\end{aligned}
$$

where the remainder term $O\left(1 /(n)^{1 / 2}\right)$ holds uniformly in any finite interval $|x| \leqq R$ as $n \rightarrow \infty$. From this follows in an obvious way our first result.

Theorem 3.1. For $r>0, R>0, n \rightarrow \infty$

$$
v_{n}(x,-t)=(2)^{1 / 2} e^{x^{2} / 8 t}\left(\frac{2 n t}{e}\right)^{n / 2}\left[\cos \left((2 n+1)^{1 / 2} \frac{x}{(4 t)^{1 / 2}}-\frac{n \pi}{2}\right)+O\left(\frac{1}{(n)^{1 / 2}}\right)\right],
$$

the order relation being valid uniformly in $|x| \leqq R, t \geqq r$.

We record next several simple preliminary results.

Lemma 3.2. For $0 \leqq x<\infty, 0<\epsilon<\infty, n=1,2, \cdots$

$$
x^{n} \leqq e^{\epsilon x^{2}}\left(\frac{n}{2 e \epsilon}\right)^{n / 2} \text {. }
$$

This is proved by computing the maximum of $e^{-\epsilon x^{2}} x^{n}$.

Lemma 3.3. For $-\infty<A<\infty,-\infty<x<\infty, 0<t<\infty, 1-4 A t>0$

$$
k(x, t) * e^{A x^{2}}=\frac{e^{A x^{2} /(1-4 A t)}}{(1-4 A t)^{1 / 2}} .
$$

This follows from the familiar addition formula

$$
k\left(x, t_{1}\right) * k\left(x, t_{2}\right)=k\left(x, t_{1}+t_{2}\right) \quad t_{1}>0, t_{2}>0 .
$$

See, for example, I. I. Hirschman and D. V. Widder [10, p. 177].

Theorem 3.4. If $-\infty<x<\infty, 0 \leqq t<\infty, 0<\delta<\infty, n=1,2, \cdots$.

$$
\left|v_{n}(x, t)\right| \leqq\left(1+\frac{t}{\delta}\right)^{1 / 2}\left[\frac{2 n(t+\delta)}{e}\right]^{n / 2} e^{x^{2} / 4 \delta} .
$$

From Lemma 3.2 and 3.3 we obtain

$$
\left|v_{n}(x, t)\right| \leqq k(x, t) *|x|^{n} \leqq\left(\frac{n}{2 e \epsilon}\right)^{n / 2} \frac{e^{\epsilon x^{2} /(1-4 \epsilon t)}}{(1-4 \epsilon t)^{1 / 2}}, \quad 0 \leqq t<1 / 4 \epsilon .
$$


Now choose $\epsilon=1 /[4(t+\delta)]$ to obtain the result. This bound on $v_{n}(x, t)$ is uniform for $-\infty<x<\infty$. To obtain a similar uniform bound on $w_{n}(x, t)$ we may use known results for $H_{n}(x)$. From H. Bateman [2, p. 208], for example,

$$
\left|H_{n}(x)\right|<A e^{x^{2} / 2} 2^{n / 2}(n !)^{1 / 2} \quad-\infty<x<\infty, n=0,1, \cdots,
$$

where $A$ is a suitable constant. By use of this formula, equations (1.11) (2.1), and Sterling's formula, we obtain

Theorem 3.5. For $-\infty<x<\infty, 0<t<\infty, n=1,2, \cdots$ and a suitable constant $A$

$$
\begin{aligned}
& \left|v_{n}(x,-t)\right| \leqq A e^{x^{2} / 8 t}\left(\frac{2 n t}{e}\right)^{n / 2} n^{1 / 4} \\
& \left|w_{n}(x, t)\right| \leqq A e^{-x^{2} / 8 t}\left(\frac{2 n}{e t}\right)^{n / 2} \frac{n^{1 / 4}}{t^{1 / 2}} .
\end{aligned}
$$

4. A generating function. We turn now to the derivation of a generating function for the biorthogonal set $v_{n}(x,-t), w_{n}(x, t)$. We need a preliminary result.

Lemma 4.1. For $-\infty<x<\infty, 0<t<\infty, n=0,1,2, \cdots$

$$
v_{n}(x,-t)=\int_{-\infty}^{\infty} k(r+i x, t)(i r)^{n} d r .
$$

By Cauchy's theorem the integral (4.1) is equal to

$$
\begin{aligned}
\int_{-\infty}^{\infty} k(r, t)(x+i r)^{n} d r & =\sum_{k=0}^{[n / 2]}\left(\begin{array}{c}
n \\
2 k
\end{array}\right) x^{n-2 k} \int_{-\infty}^{\infty} k(r, t)(i r)^{2 k} d r \\
& =n ! \sum_{k=0}^{[n / 2]} \frac{x^{n-2 k}}{(n-2 k) !} \frac{(-t)^{k}}{k !} .
\end{aligned}
$$

Comparing this with (1.2) we have the result.

Theorem 4.2. If $-\infty<x<\infty,-\infty<y<\infty,-s<t<s$

$$
k(x-y, s+t)=\sum_{n=0}^{\infty} \frac{v_{n}(x, t) w_{n}(y, s)}{n ! 2^{n}} .
$$

First suppose that $0 \leqq t<s$. From (2.2)

$$
k(y-z, s)=\sum_{n=0}^{\infty} \frac{z^{n} w_{n}(y, s)}{n ! 2^{n}} .
$$

Now take the convolution of both sides of this equation (with respect to $z$ ) with the function $k(z, t)$. We obtain 


$$
k(x-y, s+t)=\sum_{n=0}^{\infty} \frac{w_{n}(y, s)}{n ! 2^{n}} \int_{-\infty}^{\infty} k(x-z, t) z^{n} d z .
$$

We have used (3.3) to obtain the left hand side. By (1.3) we have (4.2) at once provided the term by term integration is valid. A sufficient condition for this is

$$
\sum_{n=0}^{\infty} \frac{\left|w_{n}(y, s)\right|}{n ! 2^{n}} \int_{-\infty}^{\infty} k(x-z, t)|z|^{n} d z<\infty .
$$

Using the results of $\S 3$ we see that series (4.4) is dominated by

$$
A\left(1+\frac{t}{\delta}\right)^{1 / 2} e^{x^{2} / 4 \delta} e^{-y^{2} / 8 s} \sum_{n=0}^{\infty} \frac{n^{1 / 4}}{n ! 2^{n}}\left(\frac{2 n}{e s}\right)^{n / 2}\left(\frac{2 n(t+\delta)}{e}\right)^{n / 2}
$$

for any $\delta>0$. The latter series converges for $t+\delta<s$, as one sees by the test ratio for example. Since $\delta$ is arbitrary our result is proved for $0 \leqq t<s$. The proof is not valid for negative $t$ since the addition formula (3.3) fails if $t_{1}$ or $t_{2}$ is negative.

For $t<0$ we employ the following addition formula:

$$
\int_{-\infty}^{\infty} k(z+i x, t) k(y-i z, s) d z=k(x-y, s-\imath),
$$

valid for $-\infty<x<\infty,-\infty<y<\infty, 0<t<s$. Compare I. I. Hirschman, D. V. Widder [10, p. 177]. From (4.3)

$$
k(y-i z, s)=\sum_{n=0}^{\infty} \frac{(i z)^{n}}{n ! 2^{n}} w_{n}(y, s),
$$

and by use of Lemma 4.1 the integral (4.5) becomes

$$
k(x-y, s-t)=\sum_{n=0}^{\infty} \frac{v_{n}(x,-t) w_{n}(y, s)}{n ! 2^{n}}
$$

provided

$$
\sum_{n=0}^{\infty} \frac{\left|w_{n}(y, s)\right|}{n ! 2^{n}} \int_{-\infty}^{\infty}|k(z+i x, t)||z|^{n} d z<\infty .
$$

The integral is not greater than

$$
e^{x^{2} / 4 t} \int_{-\infty}^{\infty} k(z, t)|z|^{n} d z \leqq e^{x^{2} / 4 t}\left(1+\frac{t}{\delta}\right)^{1 / 2}\left(\frac{2 n(t+\delta)}{e}\right)^{n / 2}
$$

by Theorem 3.4 with $x=0$. Note that the presence of the absolute value in (4.8) does not invalidate the estimate. Consequently the series (4.6) is dominated by 


$$
A\left(\frac{1}{t}+\frac{1}{\delta}\right)^{1 / 2} e^{x^{2} / 4 t} e^{-y^{2} / 8 s} \sum_{n=0}^{\infty} \frac{n^{1 / 4}}{n ! 2^{n}}\left(\frac{2 n}{e s}\right)^{n / 2}\left(\frac{2 n(t+\delta)}{e}\right)^{n / 2}
$$

Here we have used inequality (3.5). The latter series again converges for $0<t+\delta<s$, so that (4.7) and hence (4.6) is proved. But this is equivalent to (4.2) for negative $t$.

As a special case we could set $z^{2}=t / s$ to obtain a fundamental expansion for Hermite polynomials,

$$
\left(1-z^{2}\right)^{-1 / 2} \exp \left(y^{2}-\frac{(y-x z)^{2}}{1-z^{2}}\right)=\sum_{n=0}^{\infty} \frac{z^{n} H_{n}(x) H_{n}(y)}{2^{n} n !},
$$

valid when $|z|<1$. Compare, for example, E. C. Titchmarsh [14, p. 77].

5. Regions of convergence. We wish now to establish that the region of convergence for a series $\sum a_{n} v_{n}(x, t)$ is in general a strip $|t|<\sigma$ and for $\sum b_{n} w_{n}(x, t)$ is a half-plane $t>\sigma \geqq 0$. Let us prove some preliminary results.

Lemma 5.1. If the series

$$
\sum_{n=0}^{\infty} a_{n} v_{n}(x, t)
$$

converges at $\left(x_{0}, t_{0}\right), x_{0}>0, t_{0}>0$, then

$$
a_{n}=O\left[\left(\frac{e}{2 n t_{0}}\right)^{n / 2}\right] \quad n \rightarrow \infty .
$$

We noted in $\S 1$ that the coefficients in the expansion (1.2) are positive. Consequently when $x$ at $t$ are positive $v_{n}(x, t)$ is larger than any term of the expansion. In particular

$$
\begin{aligned}
v_{2 n}\left(x_{0}, t_{0}\right) & \geqq v_{2 n}(0, t)=\frac{(2 n) !}{n !} t_{0}^{n}, \\
v_{2 n+1}\left(x_{0}, t_{0}\right) & \geqq \frac{(2 n+1) !}{n !} x_{0} t_{0} .
\end{aligned}
$$

Since the series (5.1) is assumed to converge at $\left(x_{0}, t_{0}\right)$ the general term tends to zero as $n \rightarrow \infty$. Hence

$$
\begin{aligned}
a_{2 n}=O\left(\frac{n !}{t_{0}^{n}(2 n) !}\right)=O\left[\left(\frac{e}{4 n t_{0}}\right)^{n}\right] & n \rightarrow \infty, \\
a_{2 n+1}=O\left(\frac{n !}{t_{0}^{n}(2 n+1) !}\right)=O\left[\left(\frac{e}{(4 n+2) t_{0}}\right)^{n+1 / 2}\right] & n \rightarrow \infty .
\end{aligned}
$$

This completes the proof. Note that the lemma is no longer true if $x_{0}=0$, for then $a_{2 n+1}$ may be arbitrarily large and still have (5.1) convergent at 
$\left(0, t_{0}\right)$. This is clear since the heat polynomials of odd degree all vanish at $x=0$.

Lemma 5.2. If the series (5.1) converges for each point $(x, t)$ on the line segment $a \leqq x \leqq b, t=t_{0}<0$, then

$$
a_{n}=O\left[\left(\frac{e}{2 n\left|t_{0}\right|}\right)^{n / 2}\right]
$$$$
n \rightarrow \infty .
$$

Set

$$
c_{n}=\left(\frac{2 n \tau}{e}\right)^{n / 2}
$$$$
\tau=-t_{0}
$$

Suppose that $a_{n} c_{n}$ did not approach zero as $n \rightarrow \infty$. Then there would exist an infinite subset of positive integers $m$ for which

$$
\left|a_{m} c_{m}\right|>A>0 \text {. }
$$

By the assumed convergence of (5.1) we have for $a \leqq x \leqq b$

$$
\lim _{m \rightarrow \infty} \frac{a_{m}^{2} v_{m}^{2}\left(x, t_{0}\right)}{a_{m}^{2} c_{m}^{2}}=0 .
$$

Moreover, the quotient (5.3) is uniformly bounded on $a \leqq x \leqq b$. For, by Theorem 3.1

$$
\text { (5.4) }\left|\frac{v_{m}(x,-\tau)}{c_{m}}-(2)^{1 / 2} e^{x^{2} / 8 \tau} \cos \left((2 m+1)^{1 / 2} \frac{x}{(4 \tau)^{1 / 2}}-\frac{m \pi}{2}\right)\right| \leqq \frac{M}{(m)^{1 / 2}},
$$

where $M$ is a constant independent of $x$ in $a \leqq x \leqq b$. It is thus clear that $v_{m}\left(x, t_{0}\right) / c_{m}$ is uniformly bounded on $(a, b)$, as stated. Hence by Lebesgue's limit theorem

$$
\lim _{m \rightarrow \infty} \int_{a}^{b} \frac{v_{m}^{2}\left(x, t_{0}\right)}{c_{m}^{2}} d x=0
$$

But by (5.4) we have for $m \rightarrow \infty$

$$
\begin{aligned}
& \int_{a}^{b} \frac{v_{m}^{2}(x,-\tau)}{c_{m}^{2}} d x \\
& \quad=2 \int_{a}^{b} e^{x^{2} / 4 \tau} \cos ^{2}\left((2 m+1)^{1 / 2} \frac{x}{(4 \tau)^{1 / 2}}-\frac{m \pi}{2}\right) d x+O\left(\frac{1}{(m)^{1 / 2}}\right) \\
& \quad=\int_{a}^{b} e^{x^{2} / 4 \tau} d x+(-1)^{m} \int_{a}^{b} \cos \left(\left(\frac{2 m+1}{\tau}\right)^{1 / 2} x\right) e^{x^{2} / 4 \tau} d x+O\left(\frac{1}{(m)^{1 / 2}}\right)
\end{aligned}
$$


By the Riemann-Lebesgue theorem the second integral on the right tends to zero as $m \rightarrow \infty$. We are thus led to the contradiction

$$
\int_{a}^{b} e^{x^{2} / 4 \tau} d x=0
$$

Our assumption that $a_{n} c_{n}$ did not approach zero must have been incorrect and the lemma is proved. As in the previous lemma " $O$ " could be replaced by " $o$," but the distinction is unimportant to our purposes. This result is an analogue of the Cantor-Lebesgue theorem for trigonometric series, A. Zygmund [15, p. 267].

TheOREM 5.3. If

$$
\limsup _{n \rightarrow \infty}\left|a_{n}\right| 2 / n \frac{2 n}{e}=\frac{1}{\sigma}<\infty
$$

then the series

$$
\sum_{n=0}^{\infty} a_{n} v_{n}(x, t)
$$

converges absolutely in the strip $|t|<\sigma$ and does not converge everywhere in any including strip.

Note that this statement does not preclude the possibility of the convergence of (5.1) at certain points outside the strip $|t|<\sigma$. In fact this does occur, as we shall see. The limiting cases $\sigma=0, \sigma=\infty$ may be included in the obvious way, but let us assume that the right-hand side of (5.5) is a finite positive number.

If $0<\theta<1$, then (5.5) implies that

$$
\left|a_{n}\right| \leqq\left(\frac{e}{2 n \theta \sigma}\right)^{n / 2}
$$$$
n \geqq N
$$

for a suitable integer $N=N(\theta)$. Hence when $t>0$ the series (5.1) is dominated by

$$
e^{x^{2} / 4 \delta}\left(1+\frac{t}{\delta}\right)^{1 / 2} \sum^{\infty}\left(\frac{2 n(t+\delta)}{e}\right)^{n / 2}\left(\frac{e}{2 n \theta \sigma}\right)^{n / 2}
$$

by Theorem 3.4. But this series converges if $t+\delta<\theta \sigma$. Since $\theta$ may be taken arbitrarily near $1, \delta$ arbitrarily near 0 , the series (5.1) converges absolutely for $0 \leqq t<\sigma$.

For $t<0$ we may use (3.4). The dominant series is now

$$
A e^{-x^{2} / 8 t} \sum^{\infty}\left(\frac{2 n|t|}{e}\right)^{n / 2}\left(\frac{e}{2 n \theta \sigma}\right)^{n / 2} n^{1 / 4},
$$


and again (5.1) converges absolutely for $-\sigma<t<0$.

To prove the second part of the theorem let us suppose that (5.1) converged everywhere in the including strip $-\sigma<t<\sigma^{\prime}, \sigma^{\prime}>\sigma$, or in $-\sigma^{\prime}<t<\sigma$, $\sigma^{\prime}>\sigma$. In the former case we may apply Lemma 5.1 to obtain

$$
a_{n}=O\left[\left(\frac{e}{2 n t_{0}}\right)\right]^{n / 2} \quad n \rightarrow \infty
$$

for some $t_{0}>\sigma$. We should have as a consequence that

$$
\limsup _{n \rightarrow \infty}\left|a_{n}\right|^{2 / n} \frac{2 n}{e} \leqq \frac{1}{t_{0}}
$$

contradicting (5.5). In the latter case we could apply Lemma 5.2 with $t_{0}<-\sigma$ to obtain

$$
\begin{aligned}
a_{n} & =O\left[\left(\frac{e}{2 n\left|t_{0}\right|}\right)^{n / 2}\right], \\
\limsup _{n \rightarrow \infty}\left|a_{n}\right|{ }^{2 / n} \frac{2 n}{e} & \leqq \frac{1}{\left|t_{0}\right|},
\end{aligned}
$$

an equally definitive contradiction. The theorem is proved.

The series

$$
u(x, t)=\sum_{n=0}^{\infty} \frac{n !}{(2 n) !} v_{2 n+1}(x, t)
$$

for which $\sigma=1$ clearly converges at every point of the $t$-axis. Yet it cannot converge, according to the theorem, at every point in any strip including the strip $|t|<\sigma$. The phenomenon is a familiar one for series of homogeneous harmonic polynomials, M. Bôcher [6, p. 275].

To state the next result we must first recall the following definition; R. P. Boas [4, p. 8].

Definition 5.4. An entire function is of growth $(\rho, \tau)$ if and only if it is of order $\leqq \rho$ and is of type $\tau$ if of order $\rho$.

In the reference just cited it is shown that the function

$$
f(z)=\sum_{n=0}^{\infty} a_{n} z^{n}
$$

is of growth $(\rho, \tau)$ if and only if

$$
\limsup _{n \rightarrow \infty} \frac{n}{e \rho}\left|a_{n}\right|^{\rho / n} \leqq \tau .
$$

With this notion we can conveniently state sur next result. 
Theorem 5.5. If

$$
u(x, t)=\sum_{n=0}^{\infty} a_{n} v_{n}(x, t),
$$

the series converging for $|t|<\sigma$, then $u(x, t) \in H$ there and $u(x, 0)$ is an entire function of growth $(2,1 / 4 \sigma)$.

If $0<t_{0}<\sigma$ we have by Lemma 5.1 that

$$
a_{n}=O\left(\frac{e}{2 n t_{0}}\right)^{n / 2}
$$$$
n \rightarrow \infty \text {. }
$$

Now using Theorems 3.4 and 3.5 we see that the series (5.7) converges uniformly in any rectangle $|t| \leqq r,|x| \leqq R$ for which $r<t_{0}$. By Harnack's theorem, as applied to series of temperature functions, Täcklind [13, p. 13], the sum of the series (5.7) belongs to $H$ throughout the strip $|t|<\sigma$. Moreover, since

$$
u(x, 0)=\sum_{n=0}^{\infty} a_{n} x^{n}
$$

we find from (5.6) and (5.8) that

$$
\limsup _{n \rightarrow \infty} \frac{n}{2 e}\left|a_{n}\right|^{2 / n} \leqq \frac{1}{4 t_{0}}
$$

and that $u(x, 0)$ is entire of growth $\left(2,1 / 4 t_{0}\right)$. Allowing $t_{0}$ to approach $\sigma$ we obtain the desired result.

Corollary 5.6. For each fixed $t, 0<t<\sigma$, the series (5.7) converges uniformly in any compact region of the complex $x$-plane.

For, set $x=x^{\prime}+i x^{\prime \prime}$. Then from (1.3) we have for $\left|x^{\prime}\right| \leqq R$

$$
(4 \pi t)^{1 / 2}\left|v_{n}(x, t)\right| \leqq\left|e^{-x^{2} / 4 t}\right| \int_{-\infty}^{\infty} e^{R|y| / 2 t} e^{-y^{2} / 4 t}|y|^{n} d y .
$$

Hence in the square $\left|x^{\prime}\right| \leqq R,\left|x^{\prime \prime}\right| \leqq R$ the series (5.7) is dominated by the series of constants

$$
\frac{e^{R^{2} / t}}{(4 \pi t)^{1 / 2}} \sum_{n=0}^{\infty}\left|a_{n}\right| \int_{-\infty}^{\infty} e^{R|\nu| / 2 t} e^{-y^{2} / 4 t}|y|^{n} d y .
$$

But since $u(x, 0)$ has growth $(2,1 / 4 \sigma)$, so too do

$$
\sum_{n=0}^{\infty}\left|a_{n}\right| x^{n} \text { and } \sum_{n=0}^{\infty}\left|a_{n}\right|(-x)^{n},
$$

so that for $0<t<\sigma^{\prime}<\sigma$ 


$$
\sum_{n=0}^{\infty}\left|a_{n}\right||y|^{n}<M e^{y^{2} / 4 \sigma^{\prime}} \quad-\infty<y<\infty
$$

for a suitable constant $M$. Hence the series (5.9) converges because the integral

$$
\int_{-\infty}^{\infty} e^{R|y| / 2 t} e^{-y^{2} / 4 t} e^{y^{2} / 4 \sigma^{\prime}} d y
$$

converges. Hence by the Weierstrass $M$-test the series (5.7) converges uniformly in every square of the complex plane, as we wished to prove.

We turn next to series of associated functions.

Theorem 5.7. If

$$
\limsup _{n \rightarrow \infty} \frac{2 n}{e}\left|b_{n}\right|^{2 / n}=\sigma<\infty
$$

then the series

$$
\sum_{n=0}^{\infty} b_{n} w_{n}(x, t)
$$

converges absolutely in the half-plane $t>\sigma$ and does not converge everywhere in any including half-plane.

If $0<\theta<1$, then from $(5.10)$

$$
\left|b_{n}\right| \leqq\left(\frac{e \sigma}{2 n \theta}\right)^{n / 2}
$$$$
n \geqq N \text {. }
$$

Hence series (5.11) is dominated by

$$
A e^{-x^{2} / 8 t} \sum^{\infty}\left(\frac{e \sigma}{2 n \theta}\right)^{n / 2}\left(\frac{2 n}{e l}\right)^{n / 2} \frac{n^{1 / 4}}{t^{1 / 2}}
$$

by (3.5). But the latter series converges for $\sigma<\theta t$. Since $\theta$ is arbitrary (5.11) converges absolutely for $t>\sigma$, as stated.

To prove the concluding phrase of the theorem let us assume, contrariwise, that (5.11) converged everywhere in a half-plane including $t>\sigma$. Then

$$
\sum_{n=0}^{\infty} \frac{b_{n}}{t_{0}^{n}} v_{n}\left(x,-t_{0}\right)
$$

would converge for all $x$ and some $t_{0}<\sigma$. By Lemma 5.2

$$
b_{n}=O\left[\left(\frac{t_{0} e}{2 n}\right)^{n / 2}\right]
$$$$
n \rightarrow \infty \text {. }
$$

That is, 


$$
\sigma=\limsup _{n \rightarrow \infty} \frac{2 n}{e}\left|b_{n}\right|^{2 / n} \leqq t_{0},
$$

a contradiction. This concludes the proof. Of course it is understood that if $\sigma=0$ then the concluding phrase of the theorem becomes meaningless. For $w_{n}(x, t)$ is not defined for $t=0$ nor for $t<0$.

THEOREM 5.8. If

$$
u(x, t)=\sum_{n=0}^{\infty} b_{n} w_{n}(x, t),
$$

the series converging for $t>\sigma \geqq 0$, then $u(x, t) \in H$ there.

The proof is similar to that of Theorem 5.5 and is omitted.

6. An integral representation of temperature functions. We give next a characterization of temperature functions which have a Poisson-Stieltjes integral representation

$$
u(x, t)=\int_{-\infty}^{\infty} k(x-y, t) d \alpha(y) .
$$

A variety of necessary and sufficient conditions on $u(x, t)$ has been given to insure (6.1) with $\alpha(y)$ belonging to one or another class of functions. See, for example, I. I. Hirschman and D. V. Widder [10, pp. 17C-209]. However, the class that we shall need here seems not to have been treated. A closely related class was treated by P. C. Rosenbloom [12, p. 196].

THEOREM 6.1. The conditions

$$
\begin{array}{cc}
u(x, t) \in H & 0<t<c, \\
\int_{-\infty}^{\infty}|u(x, t)| k(x, c-t) d x<M & 0<t<c
\end{array}
$$

are necessary and sufficient that (6.1) should hold, where

$$
\int_{-\infty}^{\infty} k(x, c)|d \alpha(x)|<\infty \text {. }
$$

First to prove the conditions necessary assume (6.1) (6.4). Then (6.2) is a known property of any convergent Poisson integral, I. I. Hirschman, D. V. Widder $[10$, p. 181$]$. Also

$$
\begin{aligned}
\int_{-\infty}^{\infty}|u(x, t)| k(x, c-t) d x \leqq \int_{-\infty}^{\infty} k(x, c-t) d x \int_{-\infty}^{\infty} k(x-y, t)|d \alpha(y)| \\
=\int_{-\infty}^{\infty}|d \alpha(y)| \int_{-\infty}^{\infty} k(x, c-t) k(x-y, t) d x=\int_{-\infty}^{\infty} k(y, c)|d \alpha(y)| .
\end{aligned}
$$


We have used (3.3) and Fubini's theorem, surely valid in the presence of (6.4).

To prove the converse observe first that

$$
A y^{2}-\frac{(y-x)^{2}}{4 t} \leqq \frac{A x^{2}}{1-4 A t}
$$

for $-\infty<A<\infty,-\infty<x<\infty,-\infty<y<\infty, 0<t<\infty, 0<1-4 A t$. This is equivalent to the statement that

$$
\frac{1}{4 t}\left(y(1-4 A t)^{1 / 2}-\frac{x}{(1-4 A t)^{1 / 2}}\right)^{2} \geqq 0 .
$$

Now for $h>0$ set

$$
w_{h}(x, t)=\int_{-\infty}^{\infty} k(x-y, h) u(y, t) d y .
$$

By (6.3) this integral converges absolutely when $0<t<c-h$. Moreover, by (6.5) we have in that interval

$$
\left|w_{h}(x, t)\right| \leqq\left(\frac{c-t}{h}\right)^{1 / 2} e^{x^{2} / 4(c-t-h)} \int_{-\infty}^{\infty}|u(y, t)| k(y, c-t) d y
$$

or

$$
\left|w_{h}(x, t)\right| \leqq K e^{x^{2} / 4 \epsilon}, \quad 0<t<c-h-\epsilon .
$$

Here $K$ is independent of $t$ but may depend on $\epsilon$ and $h$.

We now employ a result which is a consequence of Tychonoff's theorem,

I. I. Hirschman and D. V. Widder [10, p. 183]. Let us state it as

LEMMA 6.2. If

$$
\begin{array}{clrl}
u(x, t) & \in H & & a<t<b, \\
|u(x, t)| & \leqq K e^{A x^{2}}, & a<t<b,
\end{array}
$$

then

$$
u(x, t)=\int_{-\infty}^{\infty} k\left(x-y, t-t^{\prime}\right) u\left(y, t^{\prime}\right) d y,
$$

the equality holding over that part of $t^{\prime}<t<b$ for which (6.11) converges absolutely.

Assume that (6.11) converges absolutely for $t^{\prime}<t<\rho \leqq b$ and denote its value by $v(x, t)$. Hence for any point $\left(x_{0}, t_{0}\right)$ of this strip and for $t^{\prime}<t<t_{0}$ 


$$
\begin{aligned}
|v(x, t)| & \leqq \int_{-\infty}^{\infty} \frac{k\left(x-y, t-t^{\prime}\right)}{k\left(x_{0}-y, t_{0}-t^{\prime}\right)}\left|u\left(y, t^{\prime}\right)\right| k\left(x_{0}-y, t_{0}-t^{\prime}\right) d y \\
& \leqq\left(\frac{t_{0}-t^{\prime}}{t-t^{\prime}}\right)^{1 / 2} e^{\left(x_{0}-x\right)^{2} / 4\left(t_{0}-t\right)} \int_{-\infty}^{\infty} k\left(x_{0}-y, t_{0}-t^{\prime}\right)\left|u\left(y, t^{\prime}\right)\right| d y .
\end{aligned}
$$

Here we have applied inequality (6.5). Hence $v(x, t)$ satisfies a uniform order condition of type (6.9) in $t^{\prime}<t<\rho-\delta, \delta>0$. (Choose $t_{0}=\rho-(\delta / 2)$, for example.) Now apply Tychonoff's uniqueness theorem to $u(x, t)-v(x, t)$. The difference satisfies (6.9) and tends to zero as $t \rightarrow t^{\prime}+$ and hence vanishes identically in $t^{\prime}<t<\rho-\delta$. Since $\delta$ is arbitrary, the lemma is proved. Note that (6.11) will certainly converge absolutely for $t<t^{\prime}+(1 / 4 A)$ by (6.9) but may do so in a larger region $[A>0]$.

By (6.7) we are in a position to apply the lemma to $w_{h}(x, t)$ in the interval $0<t<c-h-\epsilon$ and conclude

$$
w_{h}(x, t+\delta)=\int_{-\infty}^{\infty} k(x-y, t) w_{h}(y, \delta) d y, \quad 0<\delta<:+\delta<c-h-\epsilon .
$$

The integral (6.12) converges for $\delta<t+\delta<c-h$ by virtue of (6.6) with $t=\delta$.

Now allow $h$ to approach zero. By the fundamental property of the Poisson integral defining $w_{h}(x, t)$ the left hand side of (6.12) tends to $u(x, t+\delta)$. The right hand side is equal to

$$
\int_{-\infty}^{\infty} k(x-y, t) d y \int_{-\infty}^{\infty} k(y-z, h) u(z, \delta) d z=\int_{-\infty}^{\infty} k(x-z, t+h) u(z, \delta) d z
$$

provided

$$
\int_{-\infty}^{\infty} k(x-z, t+h)|u(z, \delta)| d z<\infty .
$$

Multiplying and dividing the integrand of $(6.14)$ by $k(z, c-\delta)$ and using (6.3) and (6.5) we see that the integral (6.14) is finite if $0<t+h<c-\delta$. That is, (6.13) is valid there so that we may use the right hand side of (6.13) to obtain the desired limit, $h \rightarrow 0$. The integral is an analytic, much less continuous, function of $h$ so that

$$
u(x, t+\delta)=\int_{-\infty}^{\infty} k(x-y, t) u(y, \delta) d y \quad 0<\delta<t+\delta<c .
$$

Now define for some positive $c^{\prime}<c$

$$
\beta_{\delta}(x)=\int_{-\infty}^{x} k\left(y, c^{\prime}\right) u(y, \delta) d y .
$$

Then 


$$
\begin{aligned}
\int_{-\infty}^{\infty}\left|d \beta_{\delta}(x)\right| & \leqq \int_{-\infty}^{\infty} k\left(y, c^{\prime}\right)|u(y, \delta)| d y \\
& \leqq\left(\frac{c-\delta}{c^{\prime}}\right)^{1 / 2} \int_{-\infty}^{\infty} k(y, c-\delta)|u(y, \delta)| e^{-y^{2} / 4 c^{\prime}} e^{y^{2} / 4(c-\delta)} d y \\
& \leqq M\left(\frac{c-\delta}{c^{\prime}}\right)^{1 / 2}<M\left(\frac{c}{c^{\prime}}\right)^{1 / 2}, \quad \delta<c-c^{\prime} .
\end{aligned}
$$

Hence the set of functions $\beta_{\delta}(x), 0<\delta<c-c^{\prime}$, have total variation uniformly bounded, so that we may select, by the Helly theorem, a subset approaching a function $\beta(x)$ of bounded variation on $(-\infty, \infty)$. Let $\delta \rightarrow 0$ through this subset in

$$
\lim _{\delta \rightarrow 0} \int_{-\infty}^{\infty} \frac{k(x-y, t)}{k\left(y, c^{\prime}\right)} d \beta_{\delta}(y)=\lim _{\delta \rightarrow 0} u(x, t+\delta) .
$$

By the Helly-Bray theorem we obtain

$$
u(x, t)=\int_{-\infty}^{\infty} \frac{k(x-y, t)}{k\left(y, c^{\prime}\right)} d \beta(y)=\int_{-\infty}^{\infty} k(x-y, t) d \alpha(y),
$$

where

$$
\alpha(x)=\int_{-\infty}^{x} \frac{d \beta(y)}{k\left(y, c^{\prime}\right)} .
$$

Hence (6.1) is established. Inequality (6.4) holds, since

$$
\int_{-\infty}^{\infty} k\left(x, c^{\prime}\right)|d \alpha(x)|=\int_{-\infty}^{\infty}|d \beta(x)|<M\left(\frac{c}{c^{\prime}}\right)^{1 / 2} .
$$

The function $\alpha(x)$, in spite of its definition, is independent of $c^{\prime}$ by the uniqueness theorem for the representation (6.1). Allowing $c^{\prime}$ to approach $c$, we obtain (6.4). This concludes the proof of the theorem.

7. Conditionally convergent Poisson integrals. Under the conditions of Theorem 6.1 the Poisson integral (6.1) of course converges absolutely. However, such integrals may converge conditionally, as the following example shows. Take

$$
\begin{array}{rlrl}
\phi(x) & =x e^{2 x^{2}} \sin e^{x^{2}} & & 0 \leqq x<\infty, \\
& =0 & -\infty<x \leqq 0 . \\
u(x, t) & =k(x, t) * \phi(x) & \\
u(0, t) & =\frac{1}{4(\pi t)^{1 / 2}} \int_{1}^{\infty} \frac{\sin r}{r^{p}} d r, & \\
p & =\frac{1}{4 t}-1 .
\end{array}
$$


It is clear that the integral (7.2) converges absolutely for $0<t<1 / 8$ and converges conditionally for $1 / 8 \leqq t<1 / 4$.

By an integration by parts a conditionally convergent integral (7.1) may be replaced by an absolutely convergent one, from which estimates of magnitude for $u(x, t)$ may be more readily obtained. We need several preliminary results.

Lemma 7.1. If $-\infty<A<\infty,-\infty<B<\infty$, then

$$
\int_{-\infty}^{\infty} e^{-B^{2} x^{2}+A x}|x| d x \leqq e^{A^{2} / 4 B^{2}}(|A|+|B|) /|B|^{3} .
$$

This follows in an obvious way after the change of variable $2 B^{2} x-A$ $=2 B \theta$.

Lemma 7.2. If $-\infty<x<\infty,-\infty<x_{0}<\infty, 0<t<t_{0}$, then

$$
\begin{aligned}
\int_{-\infty}^{\infty} e^{-(x-y)^{2} / 4 t} e^{\left(x_{0}-y\right)^{2} / 4 t_{0}}|x-y| & \\
& \leqq M\left(t, t_{0}\right)\left(1+\left|x-x_{0}\right|\right) e^{\left(x-x_{0}\right)^{2} / 4\left(t_{0}-t\right)}
\end{aligned}
$$

(7.4) $\int_{-\infty}^{\infty} e^{-(x-y)^{2} / 4 t} e^{\left(x_{0}-y\right)^{2} / 4 t_{0}}\left|x_{0}-y\right| d y$

$$
\leqq M\left(t, t_{0}\right)\left(1+\left|x-x_{0}\right|\right) e^{\left(x-x_{0}\right)^{2} / 4\left(t_{0}-t\right)},
$$

where $M\left(t, t_{0}\right)$ is a positive continuous function of $t$ and $t_{0}$ for $0<t<t_{0}$.

The integral (7.3) is equal to

$$
e^{\left(x_{0}-x\right)^{2} / 4 t_{0}} \int_{-\infty}^{\infty} e^{-\left(t_{0}-t\right) y^{2} / 4 t t_{0}} e^{\left(x-x_{0}\right) y / 2 t_{0}}|y| d y .
$$

Apply Lemma 7.1 with

$$
A=\frac{\left(x-x_{0}\right)}{2 t_{0}}, \quad B^{2}=\frac{t_{0}-t}{4 t t_{0}} .
$$

If

$$
M\left(t, t_{0}\right)=\frac{1}{|B|^{3}} \max \left(\frac{1}{2 t_{0}}, \frac{1}{2 t},|B|\right),
$$

then

$$
\frac{|A|+|B|}{|B|^{3}} \leqq M\left(t, t_{0}\right)\left(1+\left|x-x_{0}\right|\right),
$$

and inequality (7.3) is proved. Inequality (7.4) is established in the same way; $B^{2}$ is unchanged but now 


$$
A=\frac{x-x_{0}}{2 t}
$$

However, $M\left(t, t_{0}\right)$ was defined so that (7.5) holds in either case. The continuity of $M\left(t, t_{0}\right)$ is seen from its explicit definition.

THEOREM 7.3. If the integral

$$
u(x, t)=\int_{-\infty}^{\infty} k(x-y, t) d \alpha(y)
$$

converges at $\left(x_{0}, t_{0}\right), t_{0}>0$, then for fixed $t, 0<t<t_{0}$,

$$
u(x, t)=O\left(e^{\left(x-x_{0}\right)^{2} / 4\left(t_{0}-t\right)}\left|x-x_{0}\right|\right) \quad|x| \rightarrow \infty .
$$

To prove this set

$$
\beta(y)=\int_{0}^{y} k\left(x_{0}-z, t_{0}\right) d \alpha(z) .
$$

By the convergence of $(7.6)$ at $\left(x_{0}, t_{0}\right)$

$$
|\beta(y)|<K \quad-\infty<y<\infty
$$

for some constant $K$. Integrating (7.6) by parts we obtain

$$
u(x, t)=-\int_{-\infty}^{\infty} \beta(y) \frac{k(x-y, t)}{k\left(x_{0}-y, t_{0}\right)}\left\{\frac{x-y}{2 t}-\frac{x_{0}-y}{2 t_{0}}\right\} d y .
$$

Now apply Lemma 7.2 to obtain

$$
|u(x, t)| \leqq K \frac{\left(t_{0}\right)^{1 / 2}}{t^{1 / 2}}\left(\frac{1}{2 t}+\frac{1}{2 t_{0}}\right) M\left(t, t_{0}\right)\left(1+\left|x-x_{0}\right|\right) e^{\left(x-x_{0}\right)^{2} / 4\left(t_{0}-t\right)} .
$$

This concludes the proof. Since (7.8) converges absolutely it is clear that (7.6) converges for $0<t<t_{0}$.

CoRollary 7.4. If (7.6) converges for $0<t<c$ then for any fixed $t$ in that interval

$$
u(x, t)=O\left(e^{x^{2} / 4\left(t_{0}-t\right)}\right)
$$$$
|x| \rightarrow \infty,
$$

where $t_{0}$ is any number between $t$ and $c$.

For, it is clear that if (7.7) holds for a certain $t_{0}$ then (7.10) holds for a smaller $t_{0}$. Since $t_{0}$ is arbitrary in any case we may as well use the simpler order relation (7.9). Of course the result is best when $t_{0}$ is taken near $c$.

Corollary 7.5. If (7.6) converges for $0<t<c$ and if $0<\delta<c / 2$, then 


$$
u(x, t)=O\left(e^{x^{2} / 2 \delta}\right)
$$$$
|x| \rightarrow \infty
$$

uniformly in $\delta \leqq t \leqq c-\delta$.

For, if $t_{0}$ is chosen as $c-(\delta / 2)$ in Corollary 7.4 then (7.11) is the worst of the relations (7.10) for the interval $\delta \leqq t \leqq c-\delta$, that for $t=c-\delta$. Moreover $M\left(t, t_{0}\right)$ has a finite maximum in this closed interval. Reference to (7.9) now establishes the result.

8. The Huygens principle. As pointed out by J. Hadamard [7, p. 53] this principle has been given a variety of interpretations by various authors. As applied to the heat equation in two dimensions we shall make our meaning precise by introducing the following class $H^{*}$ of temperature functions which have the Huygens property. Compare E. Hille [9, p. 388].

Definition 8.1. A function $u(x, t)$ belongs to the class $H^{*}$ in the strip $a<t<b$ if and only if $u(x, t) \in H$ there and if

$$
u(x, t)=k\left(x, t-t^{\prime}\right) * u\left(x, t^{\prime}\right),
$$

the integral converging for every $t$ and $t^{\prime}, a<t^{\prime}<t<b$.

We show first that a function having a Poisson-Stieltjes representation belongs to $H^{*}$ in the strip of absolute convergence.

THEOREM 8.1. If

$$
u(x, t)=\int_{-\infty}^{\infty} k(x-y, t) d \alpha(y),
$$

the integral converging absolutely for $0<t<c$, then $u(x, t) \in H^{*}$ there.

For, if $0<t^{\prime}<t<c$ then

$$
\begin{aligned}
\int_{-\infty}^{\infty} k\left(x-y, t-t^{\prime}\right) u\left(y, t^{\prime}\right) d y=\int_{-\infty}^{\infty} k\left(x-y, t-t^{\prime}\right) d y \int_{-\infty}^{\infty} k\left(y-z, t^{\prime}\right) d \alpha(z) \\
=\int_{-\infty}^{\infty} d \alpha(z) \int_{-\infty}^{\infty} k\left(x-y, t-t^{\prime}\right) k\left(y-z, t^{\prime}\right) d z=\int_{-\infty}^{\infty} k(x-z, t) d \alpha(z) .
\end{aligned}
$$

The interchange in the order of integration is valid by the assumed absolute convergence of (8.2), and the theorem is proved.

We show next that $u(x, t)$ defined by (8.2) belongs to $H^{*}$ even in the strip of conditional convergence.

Theorem 8.2. If $u(x, t)$ is defined by (8.2), the integral converging for $0<t<c$, then $u(x, t) \in H^{*}$ there and the integral (8.1) converges absolutely for $0<t^{\prime}<t<c$.

We may apply Lemma 6.2. By Corollary $7.5 u(x, t)$ satisfies (6.9) in $\delta<t \leqq c-\delta$. Hence we need only show that (8.1) converges absolutely for $t^{\prime}<t<c$. Choose $t_{0}$ in Corollary 7.4 so that $t<t_{0}<c$. Then 


$$
u\left(x, t^{\prime}\right)=O\left(e^{x^{2} / 4\left(t_{0}-t^{\prime}\right)}\right)
$$$$
|x| \rightarrow \infty,
$$

from which the absolute convergence of (8.1) is evident. This concludes the proof. This result shows that although only conditional convergence is postulated in the definition of $H^{*}$, absolute convergence follows automatically.

An example of a temperature function which does not enjoy the Huygens property is

$$
u(x, t)=\int_{-\infty}^{\infty} g\left(x y, t y^{2}\right) a(y) d y
$$

where

$$
\begin{aligned}
g(x, t) & =e^{x} \cos (x+2 t)+e^{-x} \cos (x-2 t), \\
a(y) & =y \exp \left(-y^{4 / 3}\right) \cos \left(3^{1 / 2} y^{4 / 3}\right) .
\end{aligned}
$$

The present authors have shown [11] that this function $u(x, t)$ belongs to $H$ over the whole plane, is not identically zero for $t>0$, yet vanishes for all $x$ when $t=0$. Hence it certainly does not belong to $H^{*}$ in $(-1, \infty)$, for example.

It is important to note that if $u(x, t) \in H^{*}$ in two adjoining strips or even in overlapping strips it need not do so in the combined regions. A case in point is J. Blackman's example [5, p. 678]. His example was the special case $-1 / 4 a=1+i$ in the following.

Set

$$
B(x, t)=k(x, t) * k(x, a)=k(x, t+a),
$$

where $a$ is a complex number, $a=\alpha+i \beta, \alpha<0, \beta \neq 0$. Then $B(x, t)$, originally defined by (8.3) for $0<t<\left(\alpha^{2}+\beta^{2}\right) /(-\alpha)$ clearly belongs to $H$ for $-\infty<t<\infty$ by analytic continuation. From its explicit form

$$
B(x, t)=O\left(e^{o(t) x^{2}}\right) \quad|x| \rightarrow \infty
$$

where

$$
g(t)=\operatorname{Re} \frac{-1}{4(t+a)} .
$$

By the continuity of $g(t)$ and the nonvanishing of $(t+a)^{1 / 2}$ for $-\infty<t<\infty$ it is clear that $B(x, t)$ satisfies a uniform condition (6.9) in any finite strip $a<t<b$. Hence by Lemma 6.2 we have

$$
B(x, t)=k\left(x, t-t^{\prime}\right)^{*} B\left(x, t^{\prime}\right)
$$

for every $t^{\prime}$, the integral converging absolutely for $t-t^{\prime}$ sufficiently small and positive. But the integral (8.4) will not usually converge for all $t>t^{\prime}$. To get a clear picture of the region of convergence of (8.4) let us use the complex $t$-plane with $t=u+i v$. We may set $x=0$. Then 


$$
B(0, t)=\frac{1}{(4 \pi(t+a))^{1 / 2}}=\int_{-\infty}^{\infty} k\left(y, t-t^{\prime}\right) B\left(y, t^{\prime}\right) d y,
$$

and the analytic function $B(0, t)$ has a singularity at $t=-a$, a point off the real axis $(\beta \neq 0)$. The integral (8.5) may be regarded as a Laplace transform in $1 /\left(t-t^{\prime}\right)$ of a simple exponential function so that its region of convergence is well known:

$$
\operatorname{Re} \frac{1}{4\left(t-t^{\prime}\right)}>g\left(t^{\prime}\right)=\operatorname{Re} \frac{-1}{4\left(t^{\prime}+a\right)} .
$$

But this is the interior or the exterior of a circle tangent to the line $u=t^{\prime}$ at $\left(t^{\prime}, 0\right)$ and passing through the point $(-\alpha,-\beta)$ according as $t^{\prime}+\alpha<0$ or $t^{\prime}+\alpha>0$. That is, if $t^{\prime}+\alpha<0$ then (8.5) converges for all real $t$ on the diameter of this circle and in no larger interval. Or if $t^{\prime}+\alpha>0$ it converges for all real $t>t^{\prime}$. Thus, for example, $B(x, t) \in H^{*}$ in $-\infty<t<-\alpha$ and in $-\alpha<t<\infty$. Or it also belongs to $H^{*}$ in the region $0<t<-\left(\alpha^{2}+\beta^{2}\right) / \alpha$ which overlaps the two former ones but it does not do so in the combined regions. More generally, for any $c>0 B(x, t) \in H^{*}$ in the interval

$$
\left(-\frac{\alpha^{2}+\beta^{2}+c \alpha}{c+\alpha}, c\right) \text {. }
$$

It is the position of the singularity of $B(0, t)$ which governs the size of the strip of convergence of (8.4).

9. A monotonic property for certain integrals. The integral (6.3) is a decreasing function of $t$. In fact we can prove this for a more general integral as follows.

Theorem 9.1. If $u(x, t) \in H^{*}$ in $a<t<b$ and if $p \geqq 1$, then the integral

$$
J_{p}(t)=\int_{-\infty}^{\infty}|u(x, t)|^{p} k(x, b-t) d x
$$

is nonincreasing in $(a, b)$ wherever it is finite.

For, by the definition of $H^{*}$

$$
u(x, t)=\int_{-\infty}^{\infty} k\left(x-y, t-t^{\prime}\right) u\left(y, t^{\prime}\right) d y \quad a<t^{\prime}<t<b .
$$

By Hölder's inequality if $p>1$ and directly if $p=1$

$$
|u(x, t)|^{p} \leqq \int_{-\infty}^{\infty} k\left(x-y, t-t^{\prime}\right)\left|u\left(y, t^{\prime}\right)\right|^{p} d y .
$$

Here we have used the fact that 


$$
\int_{-\infty}^{\infty} k\left(x-y, t-t^{\prime}\right) d y=1
$$

Hence

$$
\begin{aligned}
J_{p}(t) & \leqq \int_{-\infty}^{\infty} k(x, b-t) d x \int_{-\infty}^{\infty} k\left(x-y, t-t^{\prime}\right)\left|u\left(y, t^{\prime}\right)\right|^{p} d y \\
& =\int_{-\infty}^{\infty}\left|u\left(y, t^{\prime}\right)\right|^{p} d y \int_{-\infty}^{\infty} k(x, b-t) k\left(x-y, t-t^{\prime}\right) d x=J_{p}\left(t^{\prime}\right) .
\end{aligned}
$$

The interchange of the order of integration is valid by Fubini's theorem provided $J_{p}\left(t^{\prime}\right)$ is finite. Thus if $J_{p}\left(t^{\prime}\right)<\infty$, then $J_{p}(t)$ is finite and decreasing in $\left(t^{\prime}, b\right)$, and the proof is complete.

The counterpart of this result for $p=\infty$ is also true.

Theorem 9.2. If $u(x, t) \in H^{*}$ in $a<t<b$, then

$$
J_{\infty}(t)=\underset{-\infty<x<\infty}{\text { l.u.b. }}|u(x, t)|
$$

is nonincreasing in $a<t<b$ wherever it is finite.

From (9.2) and (9.4) we have

$$
|u(x, t)| \leqq J_{\infty}\left(t^{\prime}\right)
$$

if $J\left(t^{\prime}\right)<\infty$. Hence $J_{\infty}(t) \leqq J_{\infty}\left(t^{\prime}\right)$ as stated.

THEOREm 9.3. If $u(x, t) \in H^{*}$ in $0<t<b$ and $p \geqq 1$, then

$$
K_{p}(t)=\frac{1}{t} \int_{-\infty}^{\infty} \frac{|u(x, t)|^{p}}{k(x, t)} d x
$$

is nonincreasing there wherever it is finite.

We note first that if $0<t^{\prime}<t$ then

$$
\int_{-\infty}^{\infty} \frac{k\left(x-y, t-t^{\prime}\right)}{k(y, t)} d y=\frac{t}{t^{\prime}} \frac{1}{k\left(x, t^{\prime}\right)} .
$$

This follows easily from Lemma 3.3. Now using (9.3) we have

$$
\begin{aligned}
K_{p}(t) & \leqq \frac{1}{t} \int_{-\infty}^{\infty} \frac{d x}{k(x, t)} \int_{-\infty}^{\infty} k\left(x-y, t-t^{\prime}\right)\left|u\left(y, t^{\prime}\right)\right|^{p} d y \\
& =\frac{1}{t} \int_{-\infty}^{\infty}\left|u\left(y, t^{\prime}\right)\right|^{p} d y \int_{-\infty}^{\infty} \frac{k\left(x-y, t-t^{\prime}\right)}{k(x, t)} d x .
\end{aligned}
$$

By (9.6) this is equal to $K_{p}\left(t^{\prime}\right)$, and the result is proved.

Preliminary to the next result we record several lemmas. 
Lemma 9.4. If $\phi(x) \in C^{1}$ in $(-\infty, \infty)$ and

$$
u(x, t)=k(x, t) * \phi(x),
$$

the integral converging absolutely at $x=0, t=t_{0}$, then

$$
\phi(y)=\lim _{R \rightarrow \infty} \int_{-R}^{R} k\left(x+i y, t_{0}\right) u\left(i x, t_{0}\right) d x \quad-\infty<y<\infty .
$$

This is a known inversion formula. Compare I. I. Hirschman and D. V. Widder $[10$, p. 176] or E. Hille [8]. We apply it to the proof of the next result.

Lemma 9.5. If $u(x, t) \in H^{*}$ in $a<t<b$, then

$$
u\left(y, t^{\prime}\right)=\int_{-\infty}^{\infty} k\left(x+i y, t-t^{\prime}\right) u(i x, t) d x
$$

for $a<t^{\prime}<t<b$.

Choose a number $a^{\prime}, a<a^{\prime}<t^{\prime}$. Then

$$
u(x, t)=\int_{-\infty}^{\infty} k\left(x-y, t-a^{\prime}\right) u\left(y, a^{\prime}\right) d y \quad a^{\prime}<t<b,
$$

the integral converging absolutely by Theorem 8.2 . Hence

$$
|u(i x, t)| \leqq e^{x^{2} / 4\left(t-a^{\prime}\right)} \int_{-\infty}^{\infty} k\left(y, t-a^{\prime}\right)\left|u\left(y, a^{\prime}\right)\right| d y .
$$

Now invert the integral

$$
u(x, t)=\int_{-\infty}^{\infty} k\left(x-y, t-t^{\prime}\right) u\left(y, t^{\prime}\right) d y
$$

by Lemma 9.4. All hypotheses are satisfied. The integral (9.11) converges absolutely by Theorem 8.2. Moreover, by virtue of (9.10) the integral (9.9) converges absolutely since $4\left(t-a^{\prime}\right)>4\left(t-t^{\prime}\right)$. Hence Cauchy's principal value (9.8) is not now needed. The conclusion is (9.9), as stated.

Theorem 9.6. If $u(x, t) \in H^{*}$ in $0<t<b$ and $p \geqq 1$, then

$$
I_{p}(t)=\int_{-\infty}^{\infty}|u(i x, t)|^{p} k(x, t) d x
$$

is nondecreasing there whenever it is finite.

By Lemma 9.5

$$
u\left(i y, t^{\prime}\right)=\int_{-\infty}^{\infty} k\left(x-y, t-t^{\prime}\right) u(i x, t) d x, \quad 0<t^{\prime}<t<b .
$$


Hence

$$
\begin{aligned}
\left|u\left(i y, t^{\prime}\right)\right|^{p} & \leqq \int_{-\infty}^{\infty} k\left(x-y, t-t^{\prime}\right)|u(i x, t)|^{p} d x, \\
I_{p}\left(t^{\prime}\right) & \leqq \int_{-\infty}^{\infty} k\left(y, t^{\prime}\right) d y \int_{-\infty}^{\infty} k\left(x-y, t-t^{\prime}\right)|u(i x, t)|^{p} d x \\
& \leqq \int_{-\infty}^{\infty}|u(i x, t)|^{p} k(x, t) d x=J_{p}(t),
\end{aligned}
$$

and the result is proved.

A glance at the proof of Theorem 9.1 will show that if $u(x, t) \geqq 0$ then $J_{1}(t)$ is constant. A generalization of this result is contained in the following theorem.

Theorem 9.7. If $u(x, t) \in H^{*}$ in $a<t<b, v(x, t) \in H^{*}$ in $a<-t<b$, and if

$$
\int_{-\infty}^{\infty}|u(x, t)| d x \int_{-\infty}^{\infty} k\left(x-y, t^{\prime}-t\right)\left|v\left(y,-t^{\prime}\right)\right| d y<\infty \quad a<t<t^{\prime}<b,
$$

then

$$
\int_{-\infty}^{\infty} u(x, t) v(x,-t) d x
$$

is constant for $a<t<b$.

By the defining property of $I I^{*}$

$$
\begin{aligned}
& u(x, t)=\int_{-\infty}^{\infty} k\left(x-y, t-t^{\prime}\right) u\left(y, t^{\prime}\right) d y \quad a<t^{\prime}<t<b, \\
& v(x,-t)=\int_{-\infty}^{\infty} k\left(x-z, t^{\prime \prime}-t\right) v\left(z,-t^{\prime \prime}\right) d z \quad a<t<t^{\prime \prime}<b .
\end{aligned}
$$

The integral (9.13) is equal to

$$
\begin{aligned}
\int_{-\infty}^{\infty} d x & \int_{-\infty}^{\infty} k\left(x-y, t-t^{\prime}\right) u\left(y, t^{\prime}\right) d y \int_{-\infty}^{\infty} k\left(x-z, t^{\prime \prime}-t\right) v\left(z,-t^{\prime \prime}\right) d z \\
& =\int_{-\infty}^{\infty} u\left(y, t^{\prime}\right) d y \int_{-\infty}^{\infty} v^{\prime}\left(z,-t^{\prime \prime}\right) k\left(y-z, t^{\prime \prime}-t^{\prime}\right) d z=\int_{-\infty}^{\infty} u\left(y, t^{\prime}\right) v\left(y,-t^{\prime}\right) d y .
\end{aligned}
$$

That is, the integral (9.13) has the same value at $t$ and $t^{\prime}$. The interchange of integrals is justified by Fubini's theorem, valid in the presence of hypothesis (9.12).

CorOllaky 9.8. If $u(x, t)$ or $v(x,-t)$ is a function of one sign on $(a, b)$ hypothesis (9.12) may be replaced by the absolute convergence of (9.13). 
10. A criterion for the Huygens property. It is clear from Theorems 6.1 and 8.1 that if

$$
\int_{-\infty}^{\infty}|u(x, t)| k(x, c-t) d x<M \quad 0<t<c
$$

then $u(x, t) \in H^{*}$ on $(0, c)$. But this sufficient condition for $H^{*}$ is not necessary. It is easy to see that the second associated function $w_{1}(x, t)$, for example, belongs to $H^{*}$ on $(0, \infty)$, but for it the integral (10.1) becomes

$$
\frac{1}{t} \int_{-\infty}^{\infty} k(x, t) k(x, c-t)|x| d x=\frac{1}{\pi}\left(\frac{c^{-1 / 2}}{t}-\frac{1}{c}\right)^{1 / 2} .
$$

This function becomes infinite as $t \rightarrow 0+$ so that (10.1) cannot be satisfied for any $M$. But this example suggests modification of the condition (10.1) to make it necessary and sufficient. It is simply that (10.1) should hold in each subinterval of $0<t<c$. We introduce the following definition.

Definition 10.1. A function $u(x, t)$ belongs to class $A$ on $a<t<b$ if and only if $u(x, t) \in H$ there and there exists a positive function $M\left(a^{\prime}, b^{\prime}\right)$ defined for $a<a^{\prime}<b^{\prime}<b$ such that

$$
\int_{-\infty}^{\infty}|u(x, t)| k\left(x, b^{\prime}-t\right) d x<M\left(a^{\prime}, b^{\prime}\right) \quad a^{\prime}<t<b^{\prime} .
$$

In the above example $W_{1}(x, t) \in A$ on $0<t<\infty$, for the function $M\left(a^{\prime}, b^{\prime}\right)$ may be taken.

$$
M\left(a^{\prime}, b^{\prime}\right)=\frac{\left(b^{\prime}\right)^{-1 / 2}}{\pi}\left(\frac{1}{a^{\prime}}-\frac{1}{b^{\prime}}\right)^{1 / 2} \quad 0<a^{\prime}<b^{\prime}<\infty .
$$

This example showed that (10.1) may give trouble at the left end of the interval in which $H^{*}$ holds. There may also be trouble at the right end. For example, if

$$
u(x, t)=k(x, t) * e^{x^{2} / 4 c}
$$

we have

$$
\int_{-\infty}^{\infty}|u(x, t)| k\left(x, b^{\prime}-t\right) d x=\left(\frac{c}{c-b^{\prime}}\right)^{1 / 2} \quad 0<t<b^{\prime} .
$$

If $b^{\prime}$ were replaced by $c$, condition (1) would fail. But the function (10.3) obviously belongs to $H^{*}$ and to $A$ on $(0, c)$. Here

$$
M\left(a^{\prime}, b^{\prime}\right)=\left(\frac{c}{c-b^{\prime}}\right)^{1 / 2} \text {. }
$$

We now show the equivalence of classes $A$ and $H^{*}$. 
Theorem 10.2. The function $u(x, t) \in H^{*}$ on $a<t<b$ if and only if $u(x, t)$ $\in A$ there.

First assume $u(x, t) \in H^{*}$. Then

$$
u(x, t)=\int_{-\infty}^{\infty} k\left(x-y, t-t^{\prime}\right) u\left(y, t^{\prime}\right) d y \quad a<t^{\prime}<t<b .
$$

By Theorem 8.2 the integral (10.1) converges absolutely. Hence

$$
J_{1}(t)=\int_{-\infty}^{\infty} k\left(y, b^{\prime}-t\right)|u(y, t)| d y<\infty \quad a<t<b^{\prime}<b .
$$

By Theorem 9.1 with $p=1$ the integral (10.5) is a nonincreasing function of $t$ so that

$$
J_{1}(t) \leqq J\left(a^{\prime}\right)
$$$$
a^{\prime}<t<b^{\prime}
$$

Hence $u(x, t) \in A$ on $(a, b)$ and we may take

$$
M\left(a^{\prime}, b^{\prime}\right)=J_{1}\left(a^{\prime}\right) .
$$

Conversely, assume (10.2). By Theorem 6.1

$$
u(x, t)=\int_{-\infty}^{\infty} k\left(x-y, t-a^{\prime}\right) d \alpha(y),
$$

the integral converging absolutely in $a^{\prime}<t<b^{\prime}$. Hence by Theorem 8.1 $u(x, t) \in H^{*}$ in $a^{\prime}<t<b^{\prime}$. But since $a^{\prime}$ and $b^{\prime}$ are arbitrary the property also holds in $a<t<b$, and the theorem is proved.

It is interesting to apply this criterion for $H^{*}$ to Blackman's function $B(x, t)$ of $\S 8$. We have for arbitrary $c$

$$
\begin{aligned}
J_{1}(t) & =\int_{-\infty}^{\infty}|B(x, t)| k(x, c-t) d x=\int_{-\infty}^{\infty} \frac{k(x, c-t) e^{o(t) x^{2}} d x}{(4 \pi|t+a|)^{1 / 2}} \\
& =\frac{1}{\left[(t+\alpha)^{2}+\beta^{2}\right]^{1 / 4}[1-4(c-t) g(t)]^{1 / 2}}, \quad 1-4(c-t) g(t)>0 .
\end{aligned}
$$

Here

$$
g(t)=\operatorname{Re} \frac{-1}{4(t+a)}=-\frac{1}{4} \frac{(t+\alpha)}{(t+\alpha)^{2}+\beta^{2}} .
$$

Hence $J_{1}(t)$ is finite and $B(x, t) \in H^{*}$ when

$$
-\frac{\alpha^{2}+\beta^{2}+c \alpha}{\alpha+c}<t<c,
$$

a result established in $\$ 8$ by another method. 
For future use let us show that the functions $v_{n}(x, t)$ and $w_{n}(x, t)$ belong to $H^{*}$.

Theorem 10.3. For $n=0,1,2, \cdots$

$$
\begin{array}{rlrl}
v_{n}(x, t) & \in H^{*} & -\infty<t<\infty, \\
w_{n}(x, t) & \in H^{*} & 0<t<\infty .
\end{array}
$$

For $v_{n}(x, t)$ the result is obvious on $(0, \infty)$ by $(1.3)$ but is less easily proved for $(-\infty, \infty)$. Using (1.2) we have

$$
\int_{-\infty}^{\infty} k\left(x-y, t-t^{\prime}\right) v_{n}\left(y, t^{\prime}\right) d y
$$

$$
\begin{aligned}
& =\int_{-\infty}^{\infty} k\left(x-y, t-t^{\prime}\right) \sum_{k=0}^{[n / 2]} \frac{n !\left(t^{\prime}\right)^{k}}{k !} \frac{y^{n-2 k}}{(n-2 k) !} d y, \\
& =\sum_{k=0}^{[n / 2]} \frac{n !\left(t^{\prime}\right)^{k}}{k !(n-2 k) !} v_{n-2 k}\left(x, t-t^{\prime}\right) \quad-\infty<t^{\prime}<t<\infty .
\end{aligned}
$$

But from (1.9)

$$
v_{n-2 k}\left(x, t-t^{\prime}\right)=\frac{(n-2 k) !}{n !} \frac{\partial^{k}}{\partial t^{k}} v_{n}\left(x, t-t^{\prime}\right) .
$$

Substituting this in (10.7) that sum becomes the Taylor expansion of $v_{n}(x, t)$ so that the integral (10.6) has the proper value as required by the property $H^{*}$.

For $w_{n}(x, t)$ it is more convenient to show it of class $A$. Since $v_{n}(x, t) \in H^{*}$ on $(0, \infty)$ we have by Theorem 10.2

$$
\int_{-\infty}^{\infty}\left|v_{n}(x, t)\right| k(x,-t) d x<M \quad 0<t<c .
$$

By (2.1) we have for $0<t<c$, since $\left|v_{n}(x,-t)\right| \leqq v_{n}(x, t)$ there,

$$
\begin{aligned}
\int_{-\infty}^{\infty}\left|w_{n}(x, t)\right| k(x, c-t) d x=\frac{1}{t^{n}} \int_{-\infty}^{\infty}\left|v_{n}(x,-t)\right| k(x, t) k(x, c-t) d x \\
\leqq \frac{1}{(4 \pi t)^{1 / 2}} \frac{1}{t^{n}} \int_{-\infty}^{\infty}\left|v_{n}(x, t)\right| k(x, c-t) d x<\frac{M}{(4 \pi t)^{1 / 2} t^{n}} .
\end{aligned}
$$

Since $c$ is arbitrary $w_{n}(x, t) \in A$ in $0<t<\infty$, as required.

11. Heat polynomial expansions. We show now that the Huygens property $H^{*}$ is necessary and sufficient for the expansion of a temperature function in a convergent series of heat polynomials.

Theorem 11.1. A necessary and sufficient condition that 


$$
u(x, t)=\sum_{n=0}^{\infty} a_{n} v_{n}(x, t),
$$

the series converging for $|t|<\sigma$ is that $u(x, t) \in H^{*}$ there. The coefficients $a_{n}$ have either of the determinations

$$
\begin{aligned}
& a_{n}=\frac{u^{(n)}(0,0)}{n !}, \\
& a_{n}=\frac{1}{n ! 2^{n}} \int_{-\infty}^{\infty} u(y,-t) w_{n}(y, t) d y \quad 0<t<\sigma .
\end{aligned}
$$

Let us assume first that $u(x, t) \in H^{*}$ in $|t|<\sigma$ and prove (11.1). Then

$$
u(x, t)=\int_{-\infty}^{\infty} k\left(x-y, t-t^{\prime}\right) u\left(y, t^{\prime}\right) d y \quad-\sigma<t^{\prime}<t<\sigma .
$$

Let us choose $t^{\prime}$ negative. By Theorem 4.2

$$
k\left(x-y, t-t^{\prime}\right)=\sum_{n=0}^{\infty} \frac{v_{n}(x, t) w_{n}\left(y,-t^{\prime}\right)}{n ! 2^{n}} .
$$

Inserting this series in (11.4) and integrating term by term we obtain (11.1), (11.3). The step will be valid if

$$
\sum_{n=0}^{\infty}\left|\frac{v_{n}(x, t)}{n ! 2^{n}}\right| \int_{-\infty}^{\infty}\left|w_{n}\left(y,-t^{\prime}\right) u\left(y, t^{\prime}\right)\right| d y<\infty .
$$

Using Theorems 3.4 and 3.5 we see that the series (11.5) is dominated by

$$
\begin{aligned}
A\left(1+\frac{t}{\delta}\right)^{1 / 2} e^{x^{2} / 4 \delta} \sum_{n=0}^{\infty} \frac{1}{n ! 2^{n}}\left(\frac{2 n(t+\delta)}{e}\right)^{n / 2}\left(\frac{2 n}{e\left|t^{\prime}\right|}\right)^{n / 2} n^{1 / 4} \\
\cdot \int_{-\infty}^{\infty} e^{y^{2} / 8 t^{\prime}}\left|u\left(y, t^{\prime}\right)\right| d y
\end{aligned}
$$

for $t \geqq 0$ and by

$$
\frac{A^{2} e^{-x^{2} / 8 t}}{\left(\left|t^{\prime}\right|\right)^{1 / 2}} \sum_{n=0}^{\infty} \frac{1}{n ! 2^{n}}\left(\frac{2 n|t|}{e}\right)^{n / 2}\left(\frac{2 n}{e\left|t^{\prime}\right|}\right)^{n / 2} n^{1 / 2} \int_{-\infty}^{\infty} e^{y^{2} / 8 t^{\prime}}\left|u\left(y, t^{\prime}\right)\right| d y
$$

for $t<0$. In each case the series converges, (11.6) for $t+\delta<\left|t^{\prime}\right|,(11.7)$ for $|t|<\left|t^{\prime}\right|$ if only the integral involved is finite. Admitting this for the moment and recalling that $\delta$ may be chosen arbitrarily small we see that series (11.1) converges absolutely to $u(x, t)$ for $|t|<\left|t^{\prime}\right|$. Since $t^{\prime}$ may be chosen arbitrarily near $-\sigma$, (11.1) converges for $|t|<\sigma$ as desired.

The integral (11.6) is finite. For, choose $c$ between $-t^{\prime}$ and $\sigma$. Then the integral (11.6) is dominated by 


$$
\left(c-t^{\prime}\right)^{1 / 2} \int_{-\infty}^{\infty}\left|u\left(y, t^{\prime}\right)\right| k\left(y, c-t^{\prime}\right) d y,
$$

which is finite by Theorem 10.2 .

Since

$$
u(x, 0)=\sum_{n=0}^{\infty} a_{n} x^{n}
$$

is a power series, the first determination (11.2) of $a_{n}$ is immediate. The second, (11.3), was obtained in the course of the proof, but it remains to show that the integral (11.3) is independent of $t$ in $(0, \sigma)$. This follows from Theorem 9.7. It is applicable since $u(x, t) \in H^{*}$ on $(-\sigma, 0)$ by hypothesis and $w_{n}(x, t)$ $\in H^{*}$ on $(0, \infty)$ by Theorem 10.3. Moreover the integral (9.12) converges on $(0, \sigma)$ by comparison with the integral (11.6). This concludes the proof of the sufficiency of the condition.

Conversely, suppose now that (11.1) holds for $|t|<\sigma$. Choose a number $c, 0<c<\sigma$, and consider the integral

$$
\int_{-\infty}^{\infty} k(x-y, t+c) u(y,-c) d y .
$$

We shall prove it equal to $u(x, t)$ for $|t|<c$. Substitute the series (11.1) in the integral (11.8) to obtain

$$
\sum_{n=0}^{\infty} a_{n} \int_{-\infty}^{\infty} k(x-y, t+c) v_{n}(y,-c) d y=\sum_{n=0}^{\infty} a_{n} v_{n}(x, t)=u(x, t)
$$

provided the term by term integration is valid. We have used the fact that $v_{n}(x, t) \in H^{*}$, as proved in Theorem 10.3. It will be sufficient to show that

$$
\sum_{n=0}^{\infty}\left|a_{n}\right| \int_{-\infty}^{\infty} k(x-y, t+c)\left|v_{n}(y,-c)\right| d y<\infty \quad-c<t<c .
$$

By (3.4) series (11.9) is dominated by

$$
A \sum_{n=0}^{\infty}\left|a_{n}\right|\left(\frac{2 n c}{e}\right)^{n / 2} n^{1 / 4} \int_{-\infty}^{\infty} k(x-y, t+c) e^{y^{2} / 8 c} d y .
$$

The integral clearly converges for $-c<t<c$. By Lemma 5.1

$$
a_{n}=O\left(\left[\frac{e}{2 n t_{0}}\right]^{n / 2}\right)
$$$$
n \rightarrow \infty
$$

for any positive $t_{0}<\sigma$. Choose $t_{0}>c$ so that series (11.10) converges. Hence the integral (11.8) is equal to $u(x, t)$ for $|t|<c$, as stated. This does not quite prove the property $H^{*}$ in $(-\sigma, \sigma)$ because of the restrictions thus far imposed 
on $c$ and $t,|t|<c$. But since $u(x, t)$ is equal to the absolutely convergent Poisson integral for $|t|<c$ we have by Theorem 8.1 that $u(x, t) \in H^{*}$ on $|t|<\sigma$, as stated.

THEOREM 11.2. If

$$
u(x, t)=\sum_{n=0}^{\infty} a_{n} v_{n}(x, t),
$$

the series converging for $|t|<\sigma$, then

$$
a_{n}=\frac{1}{(2 i)^{n} n !} \int_{-\infty}^{\infty} u(i x, t) w_{n}(x, t) d x \quad 0<t<\sigma .
$$

That is, besides the real determinations (11.2), (11.3) there is also the complex one (11.13). Observe that (11.3) involves the values of $u(x, t)$ for negative $t$, that (11.13) involves the values for positive $t$.

By Theorem 11.1

$$
u(x, t)=\int_{-\infty}^{\infty} k(x-y, t) u(y, 0) d y \quad 0<t<\sigma .
$$

For any fixed $t$ in this interval the integral (11.14) is $e^{-x^{2} / 4 t}$ multiplied by a bilateral Laplace transform in $-x /(2 t)$ which converges for $-\infty<x<\infty$. It is consequently an entire function of $x$. The same is true of the sum of the series (11.12) by Corollary 5.6. Since the two functions are equal for real $x$ the expansion (11.12) must also hold for all complex $x$ and in particular

$$
u(i x, t)=\sum_{n=0}^{\infty} a_{n} v_{n}(i x, t) \quad 0<t<\sigma .
$$

By the homogeneity of $v_{n}(x, t)$ we have

$$
v_{n}(i x, t)=i^{n} v_{n}(x,-t) .
$$

From Lemma 9.5 we have

$$
u(i x, t)=\int_{-\infty}^{\infty} k\left(x-y, t^{\prime}-t\right) u\left(i y, t^{\prime}\right) d y \quad 0<t<t^{\prime}<\sigma .
$$

Now expand the kernel by Theorem 4.2,

$$
k\left(x-y, t^{\prime}-t\right)=\sum_{n=0}^{\infty} \frac{v_{n}(x,-t) w_{n}\left(y, t^{\prime}\right)}{n ! 2^{n}} .
$$

Inserting the series (11.17) in (11.16) we have

$$
u(i x, t)=\sum_{n=0}^{\infty} A_{n} v_{n}(x,-t)=\sum_{n=0}^{\infty} A_{n}(-i)^{n} v_{n}(i x, t),
$$




$$
A_{n}=\frac{1}{n ! 2^{n}} \int_{-\infty}^{\infty} u\left(i y, t^{\prime}\right) w_{n}\left(y, t^{\prime}\right) d y \quad 0<t^{\prime}<\sigma .
$$

The integral (11.19) is constant. For, by (11.16)

$$
\begin{aligned}
\int_{-\infty}^{\infty} u(i x, t) w_{n}(x, t) d x & =\int_{-\infty}^{\infty} w_{n}(x, t) d x \int_{-\infty}^{\infty} k\left(x-y, t^{\prime}-t\right) u\left(i y, t^{\prime}\right) d y \\
& =\int_{-\infty}^{\infty} u\left(i y, t^{\prime}\right) w_{n}\left(y, t^{\prime}\right) d y,
\end{aligned}
$$

since $w_{n}(x, t) \in H^{*}$ on $(0, \infty)$. We shall see in a moment why Fubini's theorem is applicable here. If we compare coefficients in (11.18) and (11.15) we see that $A_{n}=i^{n} a_{n}$ and formula (11.13) follows. To verify the term by term integration of series (11.17) we again use Theorem 3.5 to obtain the dominant series

$$
\frac{A^{2}}{\left(t^{\prime}\right)^{1 / 2}} e^{x^{2} / 8 t} \sum_{n=0}^{\infty} \frac{1}{n ! 2^{n}}\left(\frac{2 n t}{e}\right)^{n / 2}\left(\frac{2 n}{e t^{\prime}}\right)^{n / 2} n^{1 / 2} \int_{-\infty}^{\infty} e^{-y^{2} / 8 t^{\prime}}\left|u\left(i y, t^{\prime}\right)\right| d y .
$$

By (9.10)

$$
|u(i y, t)|=O\left(e^{y^{2} / 4(t-a)}\right) \quad|y| \rightarrow \infty
$$

where $a$ may be chosen arbitrarily near to $-\sigma$. The integral (11.21) is thus seen to converge for $0<t^{\prime}<-a$ and hence for $0<t^{\prime}<\sigma$. The series (11.21) converges since $t<t^{\prime}$. Now observe that thr: convergence of the integral (11.21) is sufficient to validate the use made of Fubini's theorem in establishing (11.20). This concludes the proof of the theorem.

12. Expansions in terms of the associated functions. We now obtain two necessary and sufficient conditions, of different types, for the validity of expansions in terms of the $w_{n}(x, t)$.

Theorem 12.1. A necessary and sufficient condition that

$$
u(x, t)=\sum_{n=0}^{\infty} b_{n} w_{n}(x, t)
$$

the series converging for $t>\sigma \geqq 0$, is that

$$
u(x, t)=\frac{1}{2 \pi} \int_{-\infty}^{\infty} e^{i x y-t y^{2}} \phi(y) d y \quad \sigma<t<\infty,
$$

where $\phi(y)$ is an entire function of growth $(2, \sigma)$ and

$$
b_{n}=\frac{\phi^{(n)}(0)}{n !(-2 i)^{n}} \quad n=0,1,2, \cdots .
$$

We prove first the sufficiency of the condition (12.2). It is well known 
that the source solution $k(x, t)$ has an integral representation (12.2) with $\phi(y)=1$,

$$
k(x, t)=\frac{1}{2 \pi} \int_{-\infty}^{\infty} e^{i x y-t y^{2}} d y \quad-\infty<x<\infty, 0<t<\infty .
$$

From (2.4) we have for $n=0,1,2, \ldots$

$$
w_{n}(x, t)=\frac{1}{2 \pi} \int_{-\infty}^{\infty} e^{i x y-t y^{2}}(-2 i y)^{n} d y \quad-\infty<x<\infty, 0<t<\infty .
$$

Now expand $\phi(y)$ in power series,

$$
\phi(y)=\sum_{n=0}^{\infty} a_{n} y^{n},
$$

and substitute in (12.2), using (12.3),

$$
u(x, t)=\frac{1}{2 \pi} \int_{-\infty}^{\infty} e^{i x-t y^{2}} \sum_{n=0}^{\infty} a_{n} y^{n} d y=\sum_{n=0}^{\infty} \frac{a_{n}}{(-2 i)^{n}} w_{n}(x, t) .
$$

The operation will be valid if

$$
\int_{-\infty}^{\infty} e^{-t y^{2}} \sum_{n=0}^{\infty}\left|a_{n}\right||y|^{n} d y<\infty
$$

Since $\phi(y)$ has growth $(2, \sigma)$ the same is true of

$$
\sum_{n=0}^{\infty}\left|a_{n}\right| y^{n} \quad \text { or } \quad \sum_{n=0}^{\infty}\left|a_{n}\right|(-y)^{n}
$$

from which it follows that

$$
\sum_{n=0}^{\infty}\left|a_{n}\right||y|^{n}<M e^{\sigma^{\prime} y^{2}} \quad-\infty<y<\infty
$$

for any $\sigma^{\prime}>\sigma$ and a suitable constant $M$. Hence the integral (12.4) is dominated by

$$
M \int_{-\infty}^{\infty} e^{-t y^{2}+\sigma^{\prime} y^{2}} d y
$$

which converges for $t>\sigma^{\prime}$. Hence (12.1) is valid for $t>\sigma$ and

$$
b_{n}=\frac{a_{n}}{(-2 i)^{n}}=\frac{\phi^{(n)}(0)}{n !(-2 i)^{n}} .
$$

For the converse assume the expansion (12.1). Choose $c>\sigma$. Since the series 


$$
\sum_{n=0}^{\infty} b_{n} v_{n}(x,-t) t^{-n}
$$

converges for all $x$ on the line $t=c$ we may apply Lemma 5.2 to obtain

$$
b_{n}=O\left(\left[\frac{c e}{2 n}\right]^{n / 2}\right)
$$$$
n \rightarrow \infty \text {. }
$$

Now replace $w_{n}(x, t)$ by the integral (12.3) in the series (12.1) to obtain

$$
u(x, t)=\frac{1}{2 \pi} \sum_{n=0}^{\infty} b_{n} \int_{-\infty}^{\infty} e^{i x y-t y^{2}}(-2 i y)^{n} d y=\frac{1}{2 \pi} \int_{-\infty}^{\infty} e^{i x y-t y^{2}} \phi(y) d y,
$$

where

$$
\phi(y)=\sum_{n=0}^{\infty} b_{n}(-2 i y)^{n}=\sum_{n=0}^{\infty} a_{n} y^{n} .
$$

To justify the formal work we show that

$$
\int_{-\infty}^{\infty} e^{-t y^{2}} \sum_{n=0}^{\infty}\left|b_{n}\right||2 y|^{n} d y<\infty .
$$

By (12.5)

$$
\limsup _{n \rightarrow \infty} \frac{n\left|a_{n}\right|^{2 / n}}{2 e} \leqq c .
$$

That is, $\phi(y)$ is of growth $(2, c)$ for every $c>\sigma$ and hence also of growth $(2, \sigma)$. Hence as before the integral (12.7) converges for $t>\sigma$, and $u(x, t)$ has the representation (12.2) as desired.

Our second criterion is more closely analogous to Theorem 11.1 . We need, however, a preliminary result.

LEMMA 12.2. If

$$
u(x, t)=\frac{1}{2 \pi} \int_{-\infty}^{\infty} e^{i x y-t y^{2}} \phi(y) d y,
$$

where $\phi(y)$ has growth $(2, \sigma)$, then for each $c>\sigma$ there is a constant $M(c)$ such that

$$
|u(x, t)| \leqq \frac{M(c)}{(t-c)^{1 / 2}} e^{-x^{2} / 4(t+c)} \quad c<t<\infty .
$$

Let us shift the path of integration in (12.8) from the real axis $\eta=0$ to the line $\eta=A$ in the complex $y$-plane, $y=\xi+i \eta$. This is possible, by Cauchy's theorem, since 


$$
\begin{aligned}
\left|\int_{0}^{A} e^{i x(R+i \eta)-t(R+i \eta)^{2}} \phi(R+i \eta) d \eta\right| & \\
& \leqq N(c) e^{-t R^{2}+c R^{2}} \int_{0}^{A} e^{-x \eta+t \eta^{2}+c \eta^{2}}|d \eta|=o(1) \quad|R| \rightarrow \infty .
\end{aligned}
$$

Here we have used the assumed order of growth for $\phi(y)$ to obtain

$$
|\phi(\xi+i \eta)| \leqq N(c) e^{c\left(\xi^{2}+\eta^{2}\right)},
$$

where $c>\sigma$ and $N(c)$ depends only on $c$.

Using the new path of integration we have

$$
\begin{aligned}
u(x, t) & =\frac{1}{2 \pi} \int_{-\infty}^{\infty} e^{i x(\xi+i A)-t(\xi+i A)^{2}} \phi(\xi+i A) d \xi, \\
|u(x, t)| & \leqq \frac{N(c)}{2 \pi} e^{-A x+A^{2} t+c A^{2}} \int_{-\infty}^{\infty} e^{-t \xi^{2}+c \xi^{2}} d \xi \quad c<t<\infty .
\end{aligned}
$$

Now choose $A$ to make the right-hand side minimum; that is

$$
A=\frac{x}{2(c+t)} .
$$

Then

$$
|u(x, t)| \leqq \frac{N(c) e^{-x^{2} / 4(t+c)}}{[4 \pi(t-c)]^{1 / 2}} \quad c<t<\infty .
$$

Defining $M(c)$ in the obvious way this inequality becomes (12.9).

We can now prove our second criterion.

Theorem 12.3. A necessary and sufficient condition that

$$
u(x, t)=\sum_{n=0}^{\infty} b_{n} w_{n}(x, t)
$$

the series converging for $t>\sigma \geqq 0$ is that $u(x, t) \in H^{*}$ there and that

$$
\int_{-\infty}^{\infty}|u(x, t)| e^{x^{2} / 8 t} d x<\infty \quad \sigma<t<\infty .
$$

The coefficients $b_{n}$ have the determination

$$
b_{n}=\frac{1}{n ! 2^{n}} \int_{-\infty}^{\infty} u(x, t) v_{n}(x,-t) d x \quad \sigma<t<\infty .
$$

Observe that for the expansion (12.10) the condition $u(x, t) \in H^{*}$ is not sufficient. For example, the function $u(x, t)=1$ belongs to $H^{*}$ in $(-\infty, \infty)$. But it has no expansion (12.10). For, if it did we should have by Theorem 12.1 


$$
1=\frac{1}{2 \pi} \int_{-\infty}^{\infty} e^{i x y-t y^{2}} \phi(y) d y .
$$

But the right hand side $\rightarrow 0$ as $x \rightarrow \infty$ by the Fourier-Lebesgue theorem, a contradiction.

Let us first prove that if condition (12.11) is added them the expansion (12.10) is valid. By the $H^{*}$ assumption

$$
u(x, t)=\int_{-\infty}^{\infty} k\left(x-y, t-t^{\prime}\right) u\left(y, t^{\prime}\right) d y \quad \sigma<t^{\prime}<t<\infty .
$$

By Theorem 4.2

$$
k\left(x-y, t-t^{\prime}\right)=\sum_{n=0}^{\infty} \frac{v_{n}\left(y,-t^{\prime}\right) w_{n}(x, t)}{n ! 2^{n}},
$$

whence (12.10) follows after a term by term integration, valid if

$$
\sum_{n=0}^{\infty} \frac{\left|w_{n}(x, t)\right|}{n ! 2^{n}} \int_{-\infty}^{\infty}\left|u\left(y, t^{\prime}\right) v_{n}\left(y,-t^{\prime}\right)\right| d y<\infty .
$$

But by (3.4), (3.5) this series is dominated by

$$
\frac{A^{2}}{t^{1 / 2}} e^{-x^{2} / 8 t} \sum_{n=0}^{\infty} \frac{1}{n ! 2^{n}}\left(\frac{2 n}{e t}\right)^{n / 2}\left(\frac{2 n t^{\prime}}{e}\right)^{n / 2} n^{1 / 2} \int_{-\infty}^{\infty}\left|u\left(y, t^{\prime}\right)\right| e^{y^{2} / 8 t^{\prime}} d y .
$$

By the hypothesis (12.11) the integral (12.13) converges for $t^{\prime}>\sigma$ and the series (12.13) converges for $t^{\prime}<t$, so that the expansion (12.10) is established. The integral (12.12) is independent of $t$ by Theorem 9.7. It is applicable since $u(x, t) \in H^{*}, v_{n}(x, t) \in H^{*}$ for $\sigma<t<\infty$ and

$$
\begin{aligned}
& \int_{-\infty}^{\infty}|u(x, t)| d x \int_{-\infty}^{\infty} k\left(x-y, t^{\prime}-t\right) e^{y^{2} / 8 t^{\prime}} d y \\
&=\left(\frac{2 t^{\prime}}{t+t^{\prime}}\right)^{1 / 2} \int_{-\infty}^{\infty}|u(x, t)| e^{x^{2} / 4\left(t+t^{\prime}\right)} d x \leqq \int_{-\infty}^{\infty}|u(x, t)| e^{x^{2} / 8 t} d x<\infty . \\
& \sigma<t<t^{\prime} .
\end{aligned}
$$

That is, inequality (9.12) follows from (12.11).

Conversely, let us assume the validity of the expansion (12.10). By Theorem 12.1

$$
u(x, t)=\frac{1}{2 \pi} \int_{-\infty}^{\infty} e^{i x y-t y^{2}} \phi(y) d y,
$$

where $\phi(y)$ is of growth $(2, \sigma)$. By Lemma 12.2

$$
|u(x, t)| \leqq \frac{M(c)}{(t-c)^{1 / 2}} e^{-x^{2} / 4(t+c)} \quad \sigma<c<t<\infty .
$$


Hence

$$
\int_{-\infty}^{\infty}|u(x, t)| e^{x^{2} / 8 t} d x \leqq \frac{M(c)}{(t-c)^{1 / 2}} \int_{-\infty}^{\infty} e^{-x^{2}(t-c) / 8 t(t+c)} d x<\infty \quad c<t<\infty .
$$

Since $c$ is arbitrary (12.11) is proved.

That $u(x, t) \in H^{*}$ in $\sigma<t<\infty$ follows from Lemma 6.2 if we observe from (12.14) that $u(x, t)$ is uniformly bounded in the half-plane $t \geqq c+\delta$ for any $\delta>0$. That is, $u(x, t) \in H^{*}$ in $(c+\delta, \infty)$ and hence in $(\sigma, \infty)$. This completes the proof of the theorem.

13. $L^{2}$ expansions. Let us turn next to expansions in terms of the heat polynomials where the convergence is now to be taken in the $L^{2}$ sense.

TheOREM 13.1. If $u(x, t) \in H^{*}$ in $-\sigma \leqq t<0$ and if

$$
J_{2}(-\sigma)=\int_{-\infty}^{\infty}|u(x,-\sigma)|^{2} k(x, \sigma) d x<\infty,
$$

then for $-\sigma \leqq t<0$

$$
\lim _{N \rightarrow \infty} \int_{-\infty}^{\infty} k(x,-t)\left|u(x, t)-\sum_{n=0}^{N} a_{n} v_{n}(x, t)\right|^{2} d x=0
$$

and

$$
\int_{-\infty}^{\infty} k(x,-t)|u(x, t)|^{2} d x=\sum_{n=0}^{\infty} n !(2|t|)^{n}\left|a_{n}\right|^{2}
$$

where

$$
a_{n}=\frac{1}{n ! 2^{n}} \int_{-\infty}^{\infty} u(y, t) w_{n}(y,-t) d y \quad-\sigma \leqq t<0 .
$$

In other words, for each fixed $t$ in $-\sigma \leqq t<0$ the series

$$
\sum_{n=0}^{\infty} a_{n} v_{n}(x, t)
$$

converges in mean (with weight function $k(x,-t)$ ) to $u(x, t)$. Equation (13.3) is the corresponding Parseval relation.

By Theorem 9.1 with $p=2, c=0$ we see that $J_{2}(t)$ is nonincreasing in $(-\sigma, 0) . \mathrm{By}(13.1)$ it is then finite throughout the interval. Since

$$
v_{n}(x,-t)=t^{n / 2} H_{n}\left(\frac{x}{(4 t)^{1 / 2}}\right)
$$

we may apply the familiar $L^{2}$-expansion theorems for Hermite polynomials. Compare, for example, E. C. Titchmarsh [14, p. 79]. Following Titchmarsh, set for $n=0,1, \cdots$ 


$$
\psi_{n}(x)=d_{n} H_{n}(x) e^{-x^{2} / 2},
$$$$
d_{n}=\left(2^{n} n ! \pi^{1 / 2}\right)^{-1 / 2} .
$$

These functions form a complete orthonormal set (weight function 1) on $(-\infty, \infty)$. Hence we have the $L^{2}$ expansion

$$
\begin{aligned}
u\left((4 t)^{1 / 2} x,-t\right) e^{-x^{2} / 2} & \sim \sum_{n=0}^{\infty} a_{n}(t) \psi_{n}(x) & 0<t \leqq \sigma, \\
a_{n}(t) & =\int_{-\infty}^{\infty} e^{-x^{2} / 2} u\left(x(4 t)^{1 / 2},-t\right) \psi_{n}(x) d x . &
\end{aligned}
$$

That is, for $0<t \leqq \sigma$

$$
\lim _{N \rightarrow \infty} \int_{-\infty}^{\infty}\left|u\left(x(4 t)^{1 / 2},-t\right) e^{-x^{2} / 2}-\sum_{n=0}^{N} a_{n}(t) \psi_{n}(x)\right|^{2} d x=0,
$$

or by (13.6), (13.7)

$$
\lim _{N \rightarrow \infty} \int_{-\infty}^{\infty} k(x, t)\left|u(x,-t)-\sum_{n=0}^{N} a_{n}(t) v_{n}(x,-t) d_{n} t^{-n / 2}\right|^{2} d x=0 .
$$

Since

$$
w_{n}(x, t)=k(x, t) t^{-n / 2} H_{n}\left(x /(4 t)^{1 / 2}\right) \quad 0<t<\infty,
$$

the integral (13.8) becomes

$$
\begin{aligned}
a_{n}(t) & =(\pi)^{1 / 2} d_{n} t^{n / 2} \int_{-\infty}^{\infty} u(x,-t) w_{n}(x, t) d x, \\
a_{n}(t) t^{-n / 2} d_{n} & =\frac{1}{n ! 2^{n}} \int_{-\infty}^{\infty} u(x,-t) w_{n}(x, t) d x .
\end{aligned}
$$

But this integral was shown independent of $t$ in the proof of Theorem 11.1. Defining it as the constant $a_{n}$, as in (13.4), equation (13.2) is proved.

Parseval's equation is

$$
\begin{aligned}
\int_{-\infty}^{\infty}\left|u\left(x(4 t)^{1 / 2},-t\right)\right|^{2} e^{-x^{2}} d x & =\sum_{n=0}^{\infty}\left|a_{n}(t)\right|^{2}=\sum_{n=0}^{\infty}\left|a_{n}\right|{ }^{2} t^{n} d_{n}^{-2}, \\
\int_{-\infty}^{\infty}|u(x,-t)|^{2} k(x, t) d x & =\sum_{n=0}^{\infty} n !(2 t)^{n}\left|a_{n}\right|^{2},
\end{aligned}
$$

and the theorem is proved.

Under the assumption of the theorem that $u(x,-\sigma)(k(x, \sigma))^{1 / 2} \in L^{2}$, the representation

$$
u(x, t)=\int_{-\infty}^{\infty} k(x-y, t+\sigma) u(y,-\sigma) d y,
$$


valid by the $H^{*}$ hypothesis for $-\sigma<t<0$, may be used to extend the definition of $u(x, t)$ to the larger region $-\sigma<t<\sigma$. For, the integral (13.9) converges there by the Schwarz inequality,

$$
\begin{aligned}
{\left[\int_{-\infty}^{\infty} k(x-y, t+\sigma)|u(y,-\sigma)| d y\right]^{2} } & \\
& \leqq \int_{-\infty}^{\infty} \frac{k(x-y, t+\sigma)^{2}}{k(y, \sigma)} d y \int_{-\infty}^{\infty} k(y, \sigma)|u(y,-\sigma)|^{2} d y .
\end{aligned}
$$

The second integral on the right converges by (13.1), the first converges for $-\sigma<t<\sigma$. By Theorem 11.1 the series (13.5) also converges in the pointwise sense to $u(x, t)$ on $(0, \sigma)$. But it need not converge in the $L^{2}$ sense there. This should not be expected since the sequence $e^{x^{2} / 8 t} v_{n}(x, t), n=0,1, \cdots$ forms an orthogonal set on $(-\infty, \infty)$ for $t<0$, but the functions of the set do not even belong to $L^{2}$ for $t>0$. What is true for $t>0$ will appear in the following theorem.

Theorem 13.2. If $u(x, t) \in H^{*}$ in $0<t \leqq \sigma$ and if

$$
I_{2}(\sigma)=\int_{-\infty}^{\infty}|u(i x, \sigma)|^{2} k(x, \sigma) d x<\infty,
$$

then for $0<t \leqq \sigma$

$$
\lim _{N \rightarrow \infty} \int_{-\infty}^{\infty} k(x, t)\left|u(i x, t)-\sum_{n=0}^{N} a_{n} v_{n}(x, t)\right|^{2} d x=0
$$

and

$$
\begin{aligned}
\int_{-\infty}^{\infty} k(x, t)|u(i x, t)|^{2} d x & =\sum_{n=0}^{\infty} n !(2 t)^{n}\left|a_{n}\right|^{2}, \\
\int_{-\infty}^{\infty} k(x, t) u(i x, t)^{2} d x & =\sum_{n=0}^{\infty} n !(-2 t)^{n}\left|a_{n}\right|^{2},
\end{aligned}
$$

where

$$
a_{n}=\frac{1}{(2 i)^{n} n !} \int_{-\infty}^{\infty} u(i x, t) w_{n}(x, t) d x \quad 0<t \leqq \sigma .
$$

By Theorem 9.6 the finiteness of $I_{2}(t)$ at $t=\sigma$ implies its finiteness in $(0, \sigma)$, so that $u(i x, t) k(x, t)^{1 / 2} \in L^{2}$ on $(-\infty, \infty)$ for each fixed $t$ in that interval. Hence as in Theorem 13.1

$$
u\left(i x(4 t)^{1 / 2}, t\right) e^{-x^{2} / 2} \sim \sum_{n=0}^{\infty} a_{n}(t) \psi_{n}(x),
$$

where 


$$
a_{n}(t)=\int_{-\infty}^{\infty} u\left(i x(4 t)^{1 / 2}, t\right) e^{-x^{2} / 2} \psi_{n}(x) d x=\pi^{1 / 2} d_{n} t^{n / 2} \int_{-\infty}^{\infty} u(i x, t) w_{n}(x, t) d x .
$$

That is,

$$
\begin{array}{r}
\lim _{N \rightarrow \infty} \int_{-\infty}^{\infty}\left|u\left(i x(4 t)^{1 / 2}, t\right) e^{-x^{2} / 2}-\sum_{n=0}^{N} a_{n}(t) \psi_{n}(x)\right|^{2} d x=0, \\
\lim _{N \rightarrow \infty} \int_{-\infty}^{\infty} k(x, t)\left|u(i x, t)-\sum_{n=0}^{N} d_{n}(-t)^{-n / 2} a_{n}(t) v_{n}(i x, t)\right|^{2} d x=0 .
\end{array}
$$

If we define

$$
a_{n}=d_{n}(-t)^{-n / 2} a_{n}(t)=\frac{(-1)^{-n / 2}}{n ! 2^{n}} \int_{-\infty}^{\infty} u(i x, t) w_{n}(x, t) d x,
$$

then (13.15) becomes (13.10) and (13.16) becomes (13.13). It was shown in the proof of Theorem 11.2 that the integral (13.16) is constant.

Parseval's equation applied to (13.14) gives

$$
\int_{-\infty}^{\infty}\left|u\left(i x(4 t)^{1 / 2}, t\right)\right|^{2} e^{-x^{2}} d x=\sum_{n=0}^{\infty}\left|a_{n}(t)\right|^{2},
$$

or the same equation with absolute values removed. Using (13.16) and making an appropriate change of variables these equations become (13.11) and (13.12), respectively. This concludes the proof.

14. $L^{2}$ expansions in series of associated functions. We derive next results for the series

$$
u(x, t)=\sum_{n=0}^{\infty} b_{n} w_{n}(x, t)
$$

analogous to those of the previous section for series of heat polynomials.

Theorem 14.1. If $u(x, t) \in H^{*}$ for $t \geqq \sigma>0$ and if

$$
K_{2}(\sigma)=\frac{1}{\sigma} \int_{-\infty}^{\infty} \frac{|u(x, \sigma)|^{2}}{k(x, \sigma)} d x<\infty
$$

then for $t \geqq \sigma$

$$
\lim _{N \rightarrow \infty} \int_{-\infty}^{\infty} \frac{1}{k(x, t)}\left|u(x, t)-\sum_{n=0}^{N} b_{n} w_{n}(x, t)\right|^{2} d x=0
$$

and

$$
\int_{-\infty}^{\infty} \frac{|u(x, t)|^{2}}{k(x, t)} d x=\sum_{n=0}^{\infty} n !\left(\frac{2}{t}\right)^{n}\left|b_{n}\right|^{2}
$$


where

$$
b_{n}=\frac{1}{n ! 2^{n}} \int_{-\infty}^{\infty} u(x, t) v_{n}(x,-t) d x \quad \sigma \leqq t<\infty .
$$

By Theorem 9.3 inequality (14.1) implies that $K_{2}(t)<\infty$ for $\sigma<t<\infty$. That is, $|u(x, t)| / k(x, t)^{1 / 2} \in L^{2}$ on $-\infty<x<\infty$ for each fixed $t \geqq \sigma$. Hence

$$
\begin{aligned}
u\left(x(4 t)^{1 / 2}, t\right) e^{x^{2} / 2} & \sim \sum_{n=0}^{\infty} b_{n}(t) \psi_{n}(x), \\
b_{n}(t) & =\int_{-\infty}^{\infty} u\left(x(4 t)^{1 / 2}, t\right) e^{x^{2} / 2} \psi_{n}(x) d x \\
& =\frac{d_{n} t^{-n / 2}}{(4 t)^{1 / 2}} \int_{-\infty}^{\infty} u(x, t) v_{n}(x,-t) d x,
\end{aligned}
$$

or

$$
\lim _{N \rightarrow \infty} \int_{-\infty}^{\infty} \frac{1}{k(x, t)}\left|u(x, t)-(4 \pi t)^{1 / 2} \sum_{n=0}^{N} d_{n} t^{n / 2} b_{n}(t) w_{n}(x, t)\right|^{2} d x=0 .
$$

This is equivalent to (14.2) if

$$
b_{n}=(4 \pi t)^{1 / 2} b_{n}(t) d_{n} t^{n / 2} .
$$

But (14.7) and (14.6) give the integral determination (14.4) of $b_{n}$ as desired. We saw in the proof of Theorem 12.3 that the integral (14.4) is independent of $t$. Finally Parseval's relation applied to (14.5) gives

$$
\int_{-\infty}^{\infty}\left|u\left(x(4 t)^{1 / 2}, t\right)\right|^{2} e^{x^{2}} d x=\sum_{n=0}^{\infty}\left|b_{n}(t)\right|^{2}=\frac{1}{4 t(\pi)^{1 / 2}} \sum_{n=0}^{\infty} n !\left(\frac{2}{t}\right)^{n}\left|b_{n}\right|^{2} .
$$

This equation is (14.3) after a change of variable, and the proof is complete.

Note that equation (14.3) verifies the result of Theorem 9.2 that the integral $K_{2}(t)$ is nonincreasing. In fact it shows much more, namely that $K_{2}(t)$ is a completely monotonic function of $t$. In fact it is the Laplace transform of the positive function

$$
\sum_{n=0}^{\infty}\left|b_{n}\right|^{2}(2 y)^{n}
$$

15. Examples. Let us illustrate the foregoing theory by a variety of examples.

A. $u(x, t)=e^{a x+a^{2} t}$. Here $u(x, t) \in H^{*},-\infty<t<\infty$. Hence the $v$-expansion should converge for all $x$ and $t$.

$$
u(x, t)=\sum_{n=0}^{\infty} \frac{a^{n}}{n !} v_{n}(x, t)
$$$$
-\infty<t<\infty
$$ 


$$
\begin{aligned}
\int_{-\infty}^{\infty} k(x, t) u(x,-t)^{2} d x & =\int_{-\infty}^{\infty} k(x, t) e^{2 a x-2 a^{2} t} d x=e^{2 a^{2} t} & & 0<t<\infty, \\
\sum_{n=0}^{\infty} n !(2 t)^{n}\left|a_{n}\right|^{2} & =\sum_{n=0}^{\infty}(2 t)^{n} \frac{a^{2 n}}{n !}=e^{2 a^{2} t} & & 0<t<\infty, \\
\int_{-\infty}^{\infty} k(x, t) u(i x, t)^{2} d x & =e^{-2 a^{2} t}=\sum_{n=0}^{\infty} n !(-2 t)^{n}\left|a_{n}\right|^{2} & & 0<t<\infty .
\end{aligned}
$$

B. $u(x, t)=k(x-b, t)$. Here

$$
\int_{-\infty}^{\infty}|u(x, t)| e^{x^{2} / 8 t} d x<\infty \quad 0<t<\infty,
$$

so that the w-expansion should be valid there.

$$
\begin{array}{rlrl}
k(x-b, t) & =\sum_{n=0}^{\infty} \frac{b^{n}}{2^{n} n !} w_{n}(x, t) & 0<t<\infty, \\
\int_{-\infty}^{\infty} \frac{k(x-b, t)^{2}}{k(x, t)} d x & =e^{-b^{2} / 4 t} k(x, t) * e^{b x / 2 t}=e^{b^{2} / 2 t}, \\
\sum_{n=0}^{\infty} n !\left(\frac{2}{t}\right)^{n}\left|b_{n}\right|^{2} & =\sum_{n=0}^{\infty} \frac{b^{2 n}}{n !(2 t)^{n}}=e^{b^{2} / 2 t} .
\end{array}
$$

Also$$
k(x-b, t)=\frac{1}{2 \pi} \int_{-\infty}^{\infty} e^{i x y-t y^{2}} \phi(y) d y,
$$$$
\phi(y)=e^{-i b y},
$$

so that Theorem 12.1 is also checked.

C.

$$
\frac{x-b}{t} k(x-b, t)=\sum_{n=0}^{\infty} \frac{b^{n}}{n ! 2^{n}} w_{n+1}(x, t) \quad 0<t<\infty .
$$

This series may be obtained from (15.1) by differentiation. Further series may be obtained from it by successive differentiation.

D.

$$
u(x, t)=\int_{0}^{\infty} e^{-(1-t) u} \cosh x(u)^{1 / 2} d u
$$

$$
=\frac{(\pi)^{1 / 2} x}{2(1-t)^{3 / 2}} e^{x^{2} / 4(1-t)} \operatorname{erf}\left(\frac{x}{2(1-t)^{1 / 2}}\right)+\frac{1}{1-t} \quad-\infty<t<1 .
$$

Compare H. Bateman [3, p. 166, formula no. 35]. Here (15.2) $\int_{-\infty}^{\infty}|u(x, t)| k(x, c-t) d x \leqq \int_{0}^{\infty} e^{-(1-t) u} d u \int_{-\infty}^{\infty} k(x, c-t) \cosh x(u)^{1 / 2} d x$.

The inner integral is 


$$
\left.e^{(c-t) D^{2}} \cosh x(u)^{1 / 2}\right|_{x=0}=e^{(c-t) u},
$$

so that (15.2) converges for $-\infty<t<c$, if $c<1$. Hence $u(x, t) \in H^{*}$ in $(-\infty, 1)$ and the $v$-expansion is valid in $|t|<1$. Since

$$
\begin{aligned}
u^{(2 n)}(0,0) & =\int_{0}^{\infty} e^{-u} u^{n} d u=n !, & \\
u(x, t) & =\sum_{n=0}^{\infty} \frac{n !}{(2 n) !} v_{2 n}(x, t) & |t|<1 .
\end{aligned}
$$

E.

$$
\begin{array}{rlrl}
u(x, t) & =\int_{0}^{\infty} e^{-(1-t) u} \sinh x(u)^{1 / 2} d u & \\
& =\frac{(\pi)^{1 / 2} x e^{x^{2} / 4(1-t)}}{2(1-t)^{3 / 2}} & & -\infty<t<1 .
\end{array}
$$

Compare H. Bateman [3, p. 165, formula no. 34]. Since

$$
\left|\sinh x(\bar{u})^{1 / 2}\right| \leqq \cosh x(u)^{1 / 2},
$$

we see by Example $\mathrm{D}$ that the present function $u(x, t) \in H^{*}$ on $-\infty<t<1$. Here

$$
\begin{aligned}
a_{2 n+1} & =\frac{1}{(2 n+1) !} \int_{0}^{\infty} e^{-u} u^{(2 n+1) / 2} d u=\frac{\Gamma(n+3 / 2)}{\Gamma(2 n+2)} . \\
\frac{(\pi)^{1 / 2} x e^{x^{2} / 4(1-t)}}{2(1-t)^{3 / 2}}=\sum_{n=0}^{\infty} \frac{\Gamma(n+3 / 2)}{(2 n+1) !} v_{2 n+1}(x, t) & \mid t
\end{aligned}
$$

Note that this series exhibits the phenomenon mentioned in $\S 5$. When $x=0$ it converges for all $t$ (and even represents the function there), though the largest strip of convergence is $|t|<1$. The Parseval equation becomes

$$
\begin{array}{rlrl}
\int_{-\infty}^{\infty} k(x, t) u(x,-t)^{2} d x & =\frac{\pi}{4(1+t)^{3}(4 \pi t)^{1 / 2}} \int_{-\infty}^{\infty} x^{2} \exp \left(\frac{x^{2}(t-1)}{4 t(1+t)}\right) d x & \\
=\frac{\pi}{2} \frac{t}{\left(1-t^{2}\right)^{3 / 2}} & =\sum_{n=0}^{\infty} \frac{\Gamma(n+3 / 2)^{2}}{(2 n+1) !}(2 t)^{2 n+1} & 0<t<1, \\
\frac{1}{(1-t)^{3 / 2}} & =\sum_{n=0}^{\infty}(2 n+1)\left(\begin{array}{c}
2 n \\
n
\end{array}\right)\left(\frac{t}{4}\right)^{n} .
\end{array}
$$

This is a familiar binomial expansion.

F.

$$
u(x, t)=k(x, t) * e^{-x^{2}}=\frac{e^{-x^{2} /(1+4 t)}}{(1+4 t)^{1 / 2}} \quad t>-\frac{1}{4} .
$$

Here 


$$
u(x, t)=\frac{1}{2 \pi} \int_{-\infty}^{\infty} e^{i x y-t y^{2}} \phi(y) d y, \quad \phi(y)=(\pi)^{1 / 2} e^{-y^{2} / 4} .
$$

Here $\phi(y)$ is of growth $(2,1 / 4)$ so we must have a $w$-expansion for $t>1 / 4$. The coefficients are most conveniently obtained by expanding $\phi(y)$ in power series.

$$
u(x, t)=(\pi)^{1 / 2} \sum_{n=0}^{\infty} \frac{w_{2 n}(x, t)}{n ! 4^{2 n}}
$$

The Parseval relation is

$$
\frac{4 t}{\left(16 t^{2}-1\right)^{1 / 2}}=\sum_{n=0}^{\infty}\left(\begin{array}{c}
2 n \\
n
\end{array}\right)\left(\frac{1}{8 t}\right)^{2 n}
$$

which again may be checked by the binomial expansion.

G.

$$
u(x, t)=(\pi)^{1 / 2} k(x, t-(1 / 4))=\frac{e^{x^{2} /(1-4 t)}}{(4 t-1)^{1 / 2}} .
$$

Here $u(x, t)$ has the integral representation (15.3) with

$$
\phi(y)=(\pi)^{1 / 2} e^{y^{2} / 4} .
$$

Hence

$$
k\left(x, t-\frac{1}{4}\right)=\sum_{n=0}^{\infty} \frac{(-1)^{n} w_{2 n}(x, t)}{n ! 4^{2 n}}
$$

The Parseval equation gives (15.4), the same as in Example F.

H. Blackman's example. For $B(x, t)$ to belong to $H^{*}$ in $(-c, c)$ with $c$ as large as possible we need

$$
\begin{gathered}
c=\frac{\alpha^{2}+\beta^{2}+c \alpha}{c+\alpha}, \\
c^{2}=\alpha^{2}+\beta^{2}=|a|^{2} .
\end{gathered}
$$

The coefficients in the $v$-expansion are obtained from the power-series expansion of $e^{-x^{2} / 4 a}$.

$$
B(x, t)=\frac{1}{(4 \pi a)^{1 / 2}} \sum_{n=0}^{\infty}\left(\frac{-1}{4 a}\right)^{n} \frac{v_{2 n}(x, t)}{n !} \quad|t|<|a| .
$$

The region of convergence may be checked by formula (5.5).

I. $u(x, t)=k(x-y, t+s)$. Theorem 4.2 gives both the $v$-expansion and the w-expansion of this function. For the former

$$
a_{n}=\frac{w_{n}(y, s)}{n ! 2^{n}},
$$


considering $k(x-y, t+s)$ as a function of $x$ and $t$. It belongs to $H^{*}$ for $t>-s$, so the $v$-expansion must converge for $|t|<s$. The Parseval equation becomes

$$
\frac{e^{-y^{2} / 2(s+t)}}{4 \pi\left(s^{2}-t^{2}\right)^{1 / 2}}=\sum_{n=0}^{\infty} \frac{t^{n}}{n ! 2^{n}} w_{n}(y, s)^{2} \quad 0<t<s .
$$

For the w-expansion

$$
b_{n}=\frac{v_{n}(y, s)}{n ! 2^{n}} .
$$

Here equation (15.3) holds with

$$
\phi(z)=e^{-i y z-s z^{2}} .
$$

This function is of growth $(2, s)$, so that the $w$-expansion should converge for $t>s$, as seen also by Theorem 4.2. The Parseval equation is

$$
\left(\frac{t^{2}}{t^{2}-s^{2}}\right)^{1 / 2} e^{y^{2} / 2(t-s)}=\sum_{n=0}^{\infty} \frac{v_{n}(y, s)^{2}}{(2 t)^{n} n !} \quad t>s .
$$

\section{BIBLIOGRAPHY}

1. P. Appell, Sur l'équation $\partial^{2} z / \partial x^{2}-\partial z / \partial y$ et la théorie de la chaleur, J. Math Pures Appl. vol. 8 (1892) pp. 187-216.

2. H. Bateman and A. Erdèlyi, Higher transcendental functions, vol. II, 1953.

3. - - Tables of integral transforms, vol. I, 1954.

4. R. P. Boas, Entire functions, 1954.

5. J. Blackman, The inversion of solutions of the heat equation for the infinite rod, Duke Math. J. vol. 19 (1952) pp. 671-682.

6. M. Bôcher, On the regions of convergence of power-series which represent two-dimensional harmonic functions, Trans. Amer. Math. Soc., vol. 10 (1909) pp. 271-278.

7. J. Hadamard, Lectures on Cauchy's problem in linear partial differential equations, 1923.

8. E. Hille, $A$ class of reciprocal functions, Ann. of Math. vol. 27 (1926) pp. 427-468.

9. - Functional analysis and semi-groups, Amer. Math. Soc. Colloquium Publications, vol. 31, 1948.

10. I. I. Hirschman and D. V. Widder, The convolution transform, 1955.

11. P. C. Rosenbloom and D. V. Widder, A temperature function which vanishes initially, Amer. Math. Monthly vol. 65 (1958) pp. 607-609.

12. P. C. Rosenbloom, Linear equations of parabolic type with constant coefficients, Annals of Mathematics Studies, no. 33, 1954, pp. 191-200.

13. S. Täcklind, Sur les classes quasianalytiques des solutions des équations aux dérivées partielles du type parabolique, Nova Acta Soc. Sci. Upsal. Ser. IV, vol. 10 (1936) pp. 1-56.

14. E. C. Titchmarsh, The theory of Fourier integrals, 1937.

15. A. Zygmund, Trigonometrical series, 1952.

HARVARD UNIVERSITY, Cambridge, Mass. 